\title{
Inclusive and exclusive neutrino-nucleus cross sections and the reconstruction of the interaction kinematics
}

\author{
B. Bourguille, ${ }^{a}$ J. Nieves ${ }^{b}$ and F. Sánchez ${ }^{c, 1}$ \\ ${ }^{a}$ Institut de Fisica d'Altes Energies (IFAE), The Barcelona Institute of Science and Technology, \\ Universitat Autonoma de Barcelona, \\ Edifici Cn, Bellaterra (Barcelona), Spain \\ ${ }^{b}$ Instituto de Fisica Corpuscular (IFIC), Centro Mixto CSIC-Universidad de Valencia, \\ Institutos de Investigacion de Paterna, \\ Apartado 22085, E-46071 Valencia, Spain \\ ${ }^{c}$ Université de Genève, Faculté des Sciences, \\ Département de Physique Nucléaire et Corpusculaire (DPNC), \\ 24, Quai Ernest-Ansermet, CH-1211 Genève 4, Switzerland \\ E-mail: bruno.bourguille@free.fr, jmnieves@ific.uv.es, \\ federico.sancheznieto@unige.ch
}

ABSTRACT: We present a full kinematic analysis of neutrino-nucleus charged current quasielastic interactions based on the Local Fermi Gas model and the Random Phase Approximation. The model was implemented in the NEUT Monte Carlo framework, which allows us to investigate potentially measurable observables, including hadron distributions. We compare the predictions simultaneously to the most recent T2K and MINERvA charged current (CC) inclusive, $\mathrm{CC} 0 \pi$ and transverse kinematic-imbalance variable results. We pursuit a microscopic interpretation of the relevant reaction mechanisms, with the aim to achieving in neutrino oscillation experiments a correct reconstruction of the incoming neutrino kinematics, free of conceptual biasses. Such study is of the utmost importance for the ambitious experimental program which is underway to precisely determine neutrino properties, test the three-generation paradigm, establish the order of mass eigenstates and investigate leptonic CP violation.

Keywords: Phenomenological Models

ArXiv EPrint: 2012.12653

\footnotetext{
${ }^{1}$ Corresponding author.
} 


\section{Contents}

1 Introduction 1

2 CCQE 1p1h model 2

2.1 Implementation of the exclusive CCQE model in the MC 2

2.2 Local Fermi Gas and nucleon kinematics: implementation of the removal energy correction

2.3 Predictions of the final state hadron kinematics

3 Consistent implementation of the CC $2 \mathrm{p} 2 \mathrm{~h}$ model and the secondary nuclear collisions

4 Comparison to experimental data

4.1 Event simulation and data selection

4.1.1 T2K data samples

$\begin{array}{lll}\text { 4.1.2 MINERvA data samples } & 10\end{array}$

$\begin{array}{lll}4.2 & \text { Target composition } & 11\end{array}$

$\begin{array}{lll}4.3 & \text { Inclusive cross-sections } & 11\end{array}$

$\begin{array}{lll}4.4 & \text { Transverse kinematic-imbalance (TKI) variables } & 14\end{array}$

$\begin{array}{lll}4.5 & \text { Integrated cross-section } & 23\end{array}$

5 Data vs theoretical predictions in terms of the scaling variable $\quad \mathbf{2 5}$

5.1 Results for $R\left(\psi^{\prime}\right) \quad 29$

6 Conclusions $\quad 32$

A Comparison of momentum and energy transfer distributions 33

\section{Introduction}

The studies of neutrino-nucleus interactions are entering a new stage, motivated by longbaseline experimental programs, in which the statistical uncertainties will diminish and thus the nuclear effects - contributing to the systematical error - have to be kept well under control [1]. The incomplete theoretical knowledge of the neutrino-nucleus interactions influences various stages of experimental analysis. For instance, for the future Hyper-Kamiokande water Cherenkov detector [2], the method for reconstructing the neutrino energy will be mainly based on the kinematics of the outgoing muon, which is the only particle observed, assuming that the reaction-mechanism is two-body charged-current quasielastic (CCQE) dispersion on a bound nucleon. However, the energy range of the neutrino flux produced in the J-PARC facility [3], extending beyond $10 \mathrm{GeV}$, is such that other 
physical mechanisms give non negligible contributions to the cross section. In particular, multi-nucleon knockout processes (mainly driven by the excitation of two particle-two hole, $2 \mathrm{p} 2 \mathrm{~h}$, components in nuclei) should be taken into account. Since in the latter processes the interaction takes place on a pair of nucleons, the energy balance is different than in the QE case, driven by the excitation of only one nucleon (1p1h). Mismatching the signal coming from these two reaction mechanisms would lead to a bias in the energy reconstruction $[4,5]$. It is therefore crucial to properly include the $2 \mathrm{p} 2 \mathrm{~h}$ channel into the Monte Carlo (MC) event generators.

\section{CCQE 1p1h model}

We developed a full exclusive CCQE 1p1h MC event generator based on the theoretical scheme developed in [6]. The model is capable to simulate both neutrinos $\left(\nu_{\mu} n \rightarrow \mu^{-} p\right)$ and anti-neutrino $^{1}\left(\bar{\nu}_{\mu} p \rightarrow \mu^{+} n\right)$ reactions for a variety of nuclei: $\mathrm{C}, \mathrm{O}, \mathrm{Al}, \mathrm{Ti}, \mathrm{Fe}$ and $\mathrm{Ca}$. The original code $[6,7]$ provided the total cross-section for a fixed neutrino energy value, and the CCQE differential cross sections depending on the energy and solid angle of the outgoing charged lepton. The code was included in the NEUT MC generator [8]. The implemented modifications in NEUT keep all the physics of the model: Local Fermi Gas (LFG) nucleondynamics, short and long range Random Phase Approximation (RPA) correlations, Pauli blocking, lepton Coulomb corrections, ... and provide an almost fully exclusive cross-section by predicting the hadron kinematics in the first step (weak absorption of the gauge boson) of the reaction.

\subsection{Implementation of the exclusive CCQE model in the MC}

We implement the QE model of ref. [6] in NEUT MC, so that we could extract both the position of the first step interaction and full hadron kinematics. The model is almost fully exclusive since we compute the cross-section as function of the:

- radial position of the interaction in the nucleus and modulus of the target (hit) nucleon three-momentum from the LFG distribution.

- (anti-)neutrino energy.

- outgoing lepton momentum and angle.

- angle between the outgoing proton (neutron) and the transfer momentum direction (we test for Pauli blocking at the given radial position).

- The azimuthal angle of the final hadron with respect to the lepton reaction plane. This angle is generated with a flat probability in the $[-\pi, \pi]$ interval

\footnotetext{
${ }^{1}$ In general, we will refer to neutrino-induced reactions, unless it is explicitly mentioned that the discussion is about processes with anti-neutrinos.
} 

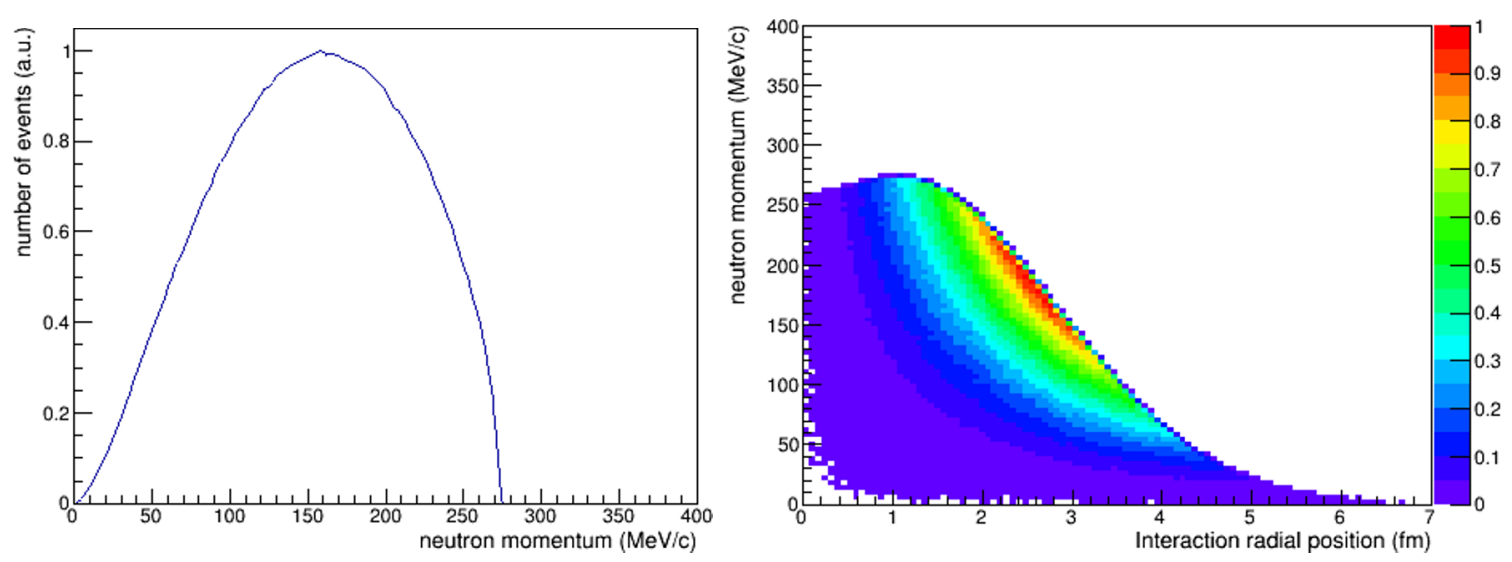

Figure 1. Left: LFG neutron momentum distribution in carbon. Right: two-dimensional neutron momentum distribution correlated to the radial interaction point inside the nucleus. Distributions are folded with the T2K $\nu$-energy flux [3].

With this information we can obtain the whole event kinematics applying conservation of momentum and energy:

- lepton four-momentum $p_{\ell}^{\mu}$.

- target (hit) nucleon four-momentum $p_{N}^{\mu}$.

- final state nucleon four-momentum $p_{N^{\prime}}^{\mu}$.

\subsection{Local Fermi Gas and nucleon kinematics: implementation of the removal energy correction}

The present model utilizes a LFG to describe the nucleus, which provides on one hand a more accurate description of the Fermi momentum and Pauli blocking than those obtained in global FG approaches. On the other hand, it allows to locate the position of the first interaction inside the nucleus, which might affect the strength/relevance of the nuclear reinteractions. We will discuss interactions of neutrinos off carbon, which is the main target material for the most recent neutrino scattering experiments: NOvA, T2K, MINERvA and MiniBooNE. Figure 1 shows that in this nucleus, the interactions mostly occur between 1.5 and $4 \mathrm{fm}$. The LFG model introduces a relation between the Fermi momentum and the radial position given by the equation:

$$
p_{F n}=\left(3 \pi^{2} \rho_{n}(r)\right)^{1 / 3}
$$

with $\rho_{n}(r)$, the density of neutrons (protons for anti-neutrino reactions) for a given radial position, $r$, inside the nucleus. In our MC, we had chosen the neutron (hit nucleon) momentum to be taken between 0 and this local Fermi momentum. The neutron momentum as function of the radial position of the interaction is shown in figure 1 . We can see that the highest local Fermi momentum is achieved at radius slightly above $1 \mathrm{fm}$ for carbon. 
At first, the model relies on the Impulse Approximation (IA), where we consider the hit-nucleon is a plane wave state, and the momentum balance reads

$$
\vec{p}_{\nu}+\vec{p}_{N}+\vec{p}_{A-1}=\vec{p}_{\mu}+\vec{p}_{N^{\prime}}+\vec{p}_{A-1}^{\prime}
$$

where $\vec{p}_{A-1}$ is the momentum of the remaining $(A-1)$-nucleons at the moment of the collision. This momentum should cancel with the hit-nucleon momentum $\left(\vec{p}_{N}\right)$ so the total momentum of the initial nucleus vanishes. Within this approximation, we also consider that the momentum of the final state nucleus $\left(\vec{p}_{A-1}^{\prime}\right)$ is equal to the residual momentum of the initial nucleus and both cancel out $\left(\vec{p}_{A-1}^{\prime}=\vec{p}_{A-1}\right)$. Thus the balance of eq. (2.2) reduces to:

$$
\vec{p}_{\nu}+\vec{p}_{N}=\vec{p}_{\mu}+\vec{p}_{N^{\prime}}
$$

which is the traditional equation of momentum conservation within the IA model. On the contrary, the IA is broken for the energy balance, due to the need of an energy contribution to transit from the ground state of the target nucleus to a new final nuclear configuration, with the daughter nucleus left in its ground or an excited state or even broken. Actually, the energy conservation equation reads

$$
E_{\nu}+M_{A}=E_{\mu}+E_{N^{\prime}}^{\infty}+M_{A-1}^{\prime}+\epsilon_{A-1}^{\prime}+T_{A-1}^{\prime}
$$

where $M_{A}$ and $M_{A-1}^{\prime}$ are the ground state masses of the initial and final nuclei, and $E_{N^{\prime}}^{\infty}$ is the energy of the asymptotically observed nucleon $\left(N^{\prime}\right)$. In addition, $\epsilon_{A-1}^{\prime}>0$ is the excitation energy of the final nucleus, which average is expected to be between 10 and $20 \mathrm{MeV}$ (see figure 2). Finally, $T_{A-1}^{\prime}$ is the final nucleus kinetic energy, which is very small (typically of the order of $p_{F_{n}}^{2} /\left(2 M_{A-1}\right) \sim 2 \mathrm{MeV}$ for carbon target) and it is approximated to zero in what follows.

The energy balance in eq. (2.4) does not apply to cases where any secondary rescattering collision changes the energy of the nucleon that emerges after the weak absorption of the gauge boson. ${ }^{2}$

The excitation energy can be estimated, in a first approximation, to be the energy of the hole, within the FG model ${ }^{3}$

$$
\epsilon_{A-1}^{\prime} \sim\left(T_{F}-T_{N}\right)
$$

with $T_{F}$ the kinetic energy of the nucleon at the Fermi level for the given radial position, and $T_{N}$ the actual kinetic energy of the knocked out nucleon in the target nucleus. On the other hand, the experimental nucleon separation energy $S_{N}$ can be obtained from the masses of the initial and final nuclei:

$$
S_{N}=M_{A-1}^{\prime}-M_{A}+m_{N}
$$

\footnotetext{
${ }^{2}$ In fact, in these latter situations, the rupture of the daughter nucleus might occur and the analysis is more complicated.

${ }^{3}$ Any LFG model implicitly assumes the existence of a mean-field potential $U=-T_{F}$, which cancels in the difference of energies, and binds the nucleons.
} 


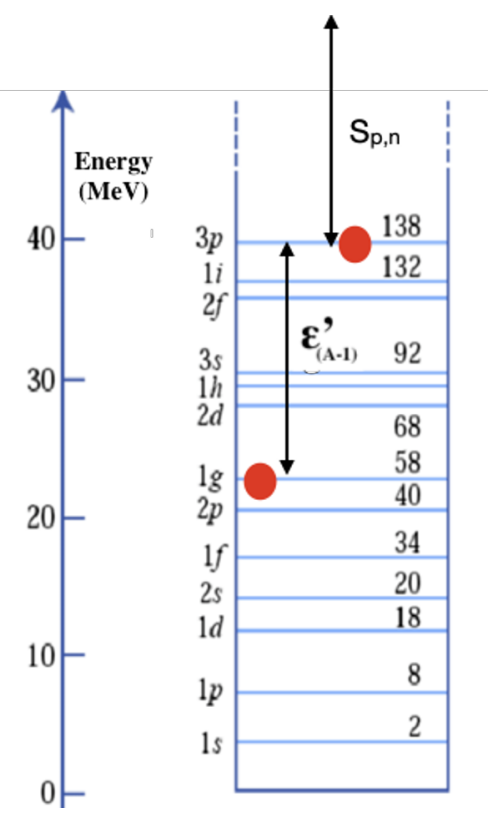

Figure 2. Pictorial representation of the excitation energy scheme. When a nucleon is removed from a deep shell, the hole energy remains until the nucleus is de-excited. The FG removal energy refers only to Fermi level nucleons, which would correspond to leave the daughter $(A-1)$ nucleus in the ground state within this statistical nuclear model. It is an approximation to the experimental proton and neutron separation energies $\left(S_{p, n}\right)$ of the target nucleus. Knocking out a nucleon from deeper levels will give rise to excited final-nucleus states, equivalent to the binding energy of the nucleon occupying this level $(\epsilon)$. We also show the accumulative occupation numbers when additional shells are considered. The energy scale depicted at the left of the plot has an arbitrary origin, and it is only intended to illustrate the energy differences between shells.

where $M_{A}$ and $M_{A-1}^{\prime}$ are the ground-state masses of the initial $\left(A_{Z}\right)$ and final $\left[(A-1)_{Z}\right.$ or $(A-1)_{Z-1}$ for neutrino or anti-neutrino reactions] nuclei, and $m_{N}$ the mass of the target nucleon. Re-writing eq. (2.4) using $S_{N}$, we obtain:

$$
E_{\nu}+\left(m_{N}+T_{N}-T_{F}\right)=E_{\mu}+S_{N}+E_{N^{\prime}}^{\infty}
$$

which reduces to the usual IA energy conservation formula, but with an additional correction: the term $S_{N}+T_{F}$, which is an approximation of the experimental removal energy and takes into account to some level the excitation of the final state nucleus. In case of the Relativistic Global Fermi Gas (RGFG), $T_{F}$ takes a constant value $(\sim 27 \mathrm{MeV})$, but in the relativistic LFG model, it has a distribution depending on the radial position of the interaction. This dependency introduces several removal energies simulating a continuous distribution of excitation of nuclear states.

This discussion of the IA energy balance fixes a problem with the nuclear missing energy $\left(E_{m}^{I A}\right)$, which appears within the traditional implementation in MC event generators of the relativistic LFG and RGFG models

$$
E_{m}^{I A}=E_{\nu}-E_{\mu}-T_{N^{\prime}}^{\infty}=S_{N}+\left(m_{N^{\prime}}-m_{N}\right)+T_{F}-T_{N}
$$



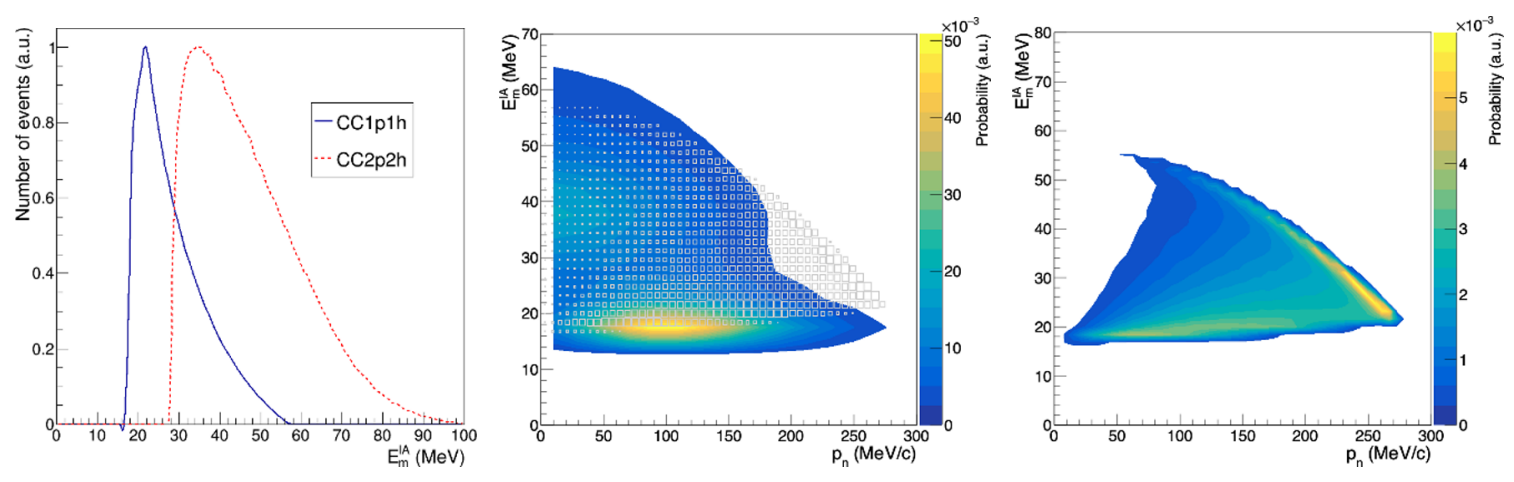

Figure 3. Left: number of events as function of the $E_{m}^{I A}$ energy for neutrino scattering off ${ }^{12} \mathrm{C}$ within the LFG model. Both the 1 p1h (eq. (2.8)) and 2p2h (eq. (3.3)) $E_{m}^{I A}$ distributions are shown by the blue-solid and red-dashed lines, respectively. The gap between the two distributions is caused by the excitation energy of the two holes in the final state. As in figure 1, results have been folded with the T2K neutrino energy flux. Center: probability to find a neutron in carbon with a momentum $\left(p_{n}\right)$ for a given reaction missing energy $\left(E_{m}^{I A}\right)$ (see eq. (2.8)) as predicted by the SF model [9-11] (contour plot) and for this implementation of the LFG (box plot). Right: LFG predictions corresponding to the box plot displayed in the middle panel. In all cases, the T2K flux [3] is used.

with $E_{N^{\prime}}^{\infty}=T_{N^{\prime}}^{\infty}+m_{N^{\prime}}$. The value of $E_{m}^{I A}$ becomes negative (non-physical) for some values of $T_{N}$ when, as it is common, the $T_{F}$ correction is not added. ${ }^{4}$ Equivalently, the problem is caused by the wrong assumption of taking $E_{N}=\left(m_{N}+T_{N}\right)$, instead of the correct expression $E_{N}=\left(m_{N}+T_{N}-T_{F}\right)$, which includes the mean field potential responsible for binding the nuclear system. The distribution $E_{m}^{I A}$ of energies for a relativistic LFG is depicted in the left plot of figure 3 for neutrino scattering off carbon, where $S_{n}+\left(m_{p}-m_{n}\right)=$ $\Delta\left({ }^{11} \mathrm{C}\right)-\Delta\left({ }^{12} \mathrm{C}\right)+\Delta\left({ }^{1} \mathrm{H}\right)=17.4 \mathrm{MeV}\left[\Delta\left(A_{Z}\right)\right.$ is the mass excess of the $A_{Z}$ nucleus $]$. The average $E_{m}^{I A}$ is $28 \mathrm{MeV}$, very similar to the binding energies used in RGFG models $(25 \mathrm{MeV})$ [14] or in MINERvA $(27.13 \mathrm{MeV})$ [15]. ${ }^{5}$ In addition, we can use this average of $E_{m}^{I A}$ for the LFG model in carbon, to estimate the average of the excitation energy $\left\langle\epsilon_{A-1}^{\prime}\right\rangle \sim\left\langle T_{F}-T_{N}\right\rangle \sim 11 \mathrm{MeV}$ for this target, using $S_{n}+\left(m_{p}-m_{n}\right)=17.4 \mathrm{MeV}$.

We pay now attention to the two-dimensional $\left(p_{N}, E_{m}^{I A}\right)$ distribution shown in the middle and right plots of figure 3 following the same representation as in the Spectral Function (SF) scheme [9-11]. The carbon SF obtained in [10] is comprised of two contributions. The first one is determined by a mean-field description of the nucleus, while the second one takes into account two-nucleon short range correlations, and it is computed within a correlated basis function scheme in isospin-symmetric nuclear matter. The mean-field contribution of the SF modifies the dispersion relation by forcing a set of effective bound masses. This way the value of $E_{m}^{I A}$ is constant for each of the nuclear levels with a broad momentum distribution, which is additionally distorted by the contribution of the correlated part of the SF. The model presented here is based on the LFG approach to the nucleus, where the

\footnotetext{
${ }^{4}$ This is taken into account in some models such as NuWro by adding a constant that restores the validity of the model.

${ }^{5}$ See also data release package.
} 

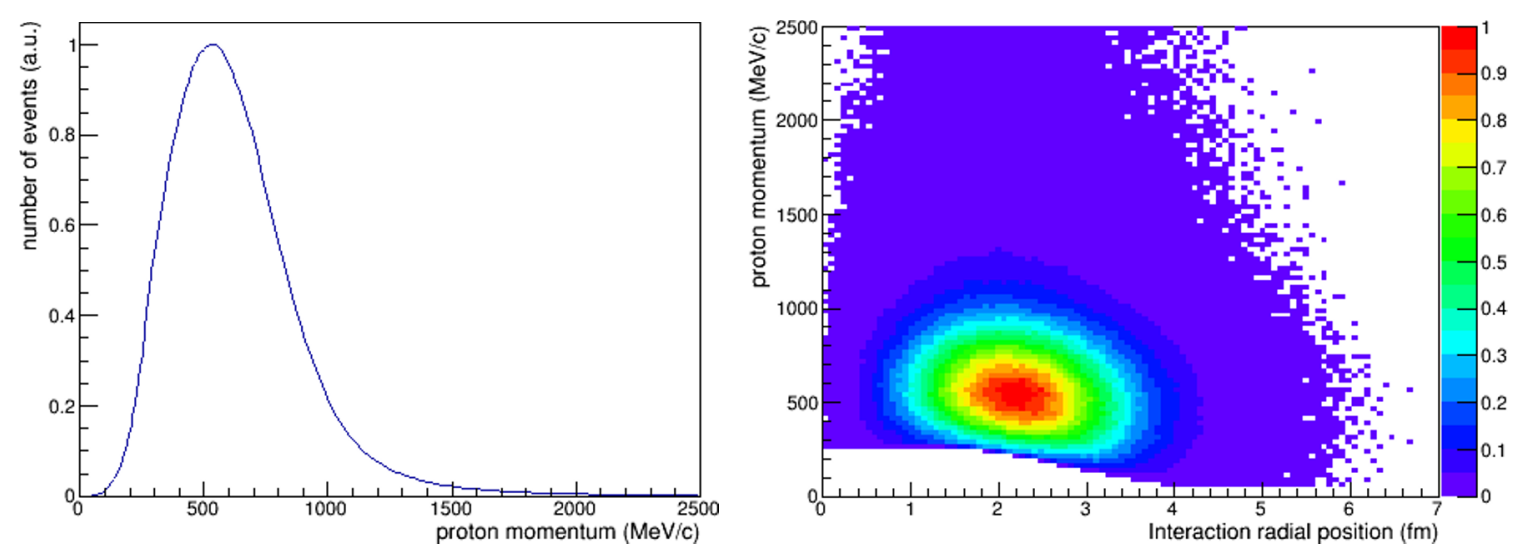

Figure 4. Left: primary proton, created after the absorption of the gauge boson $W^{+}$in neutrino processes, momentum distribution in carbon predicted by the model. Right: proton momentum distribution predicted by the model as function of the radial interaction point inside the nucleus. As in figure 1, results have been folded with the T2K neutrino energy flux.

dispersion relation is fixed to the on-shell target nucleon mass, ${ }^{6}$ but with a Fermi level that depends on the spatial position through the local density. Despite its simplicity, the LFG distribution, as shown in figure 3, follows a pattern similar to that exhibited by the more realistic one inferred from the SF scheme of refs. [9-11]. Nevertheless, some differences between both sets of predictions are visible in figure 3 , in particular at the edges of the $\left(p_{N}, E_{m}^{I A}\right)$ contours. This different dependencies might introduce distinctive differences in the nuclear response when the nucleon target momentum is relevant such as in the case of low energy neutrino interactions and it might explain some of the disagreements discussed later in this work.

\subsection{Predictions of the final state hadron kinematics}

In the left panel of figure 4 we show the momentum distribution of the primary proton, created after the absorption of the gauge boson in neutrino processes, as predicted by the model presented in this work. In the right plot of the figure, we show the proton momentum correlated to the radial position of the primary interaction. Low energy protons are produced close to the outer surface of the nucleus having a large probability to survive nuclear re-scattering. The maximum momentum of the proton is limited by the energy of the neutrino, but the lowest values are determined by Pauli blocking, which is also function of the radial position of the interaction. The fact that the Pauli effects become less relevant at large radii $(4 \mathrm{fm})$ allows the proton momentum to have values close to zero contrary to less sophisticated models such as the global FG [8].

\footnotetext{
${ }^{6}$ The $1 \mathrm{p} 1 \mathrm{~h}$ contribution to the nuclear response function depends on the energy difference between particle- and hole- nucleons, where the mean-field potential $-T_{F}(r)$ cancels out.
} 


\section{Consistent implementation of the $\mathrm{CC} 2 \mathrm{p} 2 \mathrm{~h}$ model and the secondary nuclear collisions}

In NEUT [8], the events are generated according to the distribution of the outgoing lepton, i.e. using the weight given by the value of the double-differential inclusive cross section, expressed as the contraction of lepton and hadron tensors, as given for instance in eq. (2) of ref. [7]. In addition to the $1 \mathrm{p} 1 \mathrm{~h}$ term, the hadron tensor $W^{\mu \nu}$ accounts also for $2 \mathrm{p} 2 \mathrm{~h}$ contributions evaluated following the LFG scheme of ref. [7], which is fully consistent with the $1 \mathrm{p} 1 \mathrm{~h}$ implementation outlined above and based on [6]. It is computed, for (anti)neutrino reactions, separately for proton-neutron and proton-proton (neutron-neutron) final states to provide isospin dependent final states. The location of the interaction vertex in the nucleus is chosen according to the density profile, and the initial state nucleons are picked below the Fermi level corresponding to the radial position following the LFG model recipe. The outgoing nucleons at the weak vertex are distributed according to the available phasespace. This is because all the hadron-variables are integrated out in the calculation of the inclusive lepton cross sections carried out in [7]. At this respect, note that in the recent re-computation of ref. [18] some of these integrations have been undone, opening the possibility to improve on this phase-space prescription. The final state nucleons are generated uniformly in the center of the mass of the hadronic system and boosted to the laboratory rest frame. Next, their momenta are tested against the local Fermi level to implement Pauli blocking. This procedure neglects the dynamics of underlying nuclear model and produces a symmetric distribution of outgoing nucleons [18]. The produced pair of nucleons is fed into the NEUT cascade model accounting for the transport of nucleons in the high density nuclear medium.

We have introduced the same removal energy corrections than in the case of the $1 \mathrm{p} 1 \mathrm{~h}$, but we need to take into account the fact that two nucleons are removed from the nucleus. This provides an energy balance equation:

$$
\begin{aligned}
E_{\nu}= & E_{\mu}+E_{N_{1}^{\prime}}^{\infty}+E_{N_{2}^{\prime}}^{\infty}+S_{N_{1} N_{2}} \\
& -\left(m_{N_{1}}+T_{N_{1}}-T_{F_{1}}\right)-\left(m_{N_{2}}+T_{N_{2}}-T_{F_{2}}\right)
\end{aligned}
$$

where we have neglected the kinetic energy of the daughter nucleus, have contemplated the possibility of different (isospin) Fermi levels for the hit nucleons $N_{1}$ and $N_{2}$, and

$$
S_{N_{1} N_{2}}=M_{A-2}^{\prime}-M_{A}+m_{N_{1}}+m_{N_{2}}
$$

Finally, $N_{1}^{\prime}$ and $N_{2}^{\prime}$ are the two outgoing nucleons asymptotically observed. In the left plot of figure 3 , we showed the distribution of missing energies from the $2 \mathrm{p} 2 \mathrm{~h}$ mechanism

$$
\left.E_{m}^{I A}\right|_{2 \mathrm{p} 2 \mathrm{~h}}=E_{\nu}-E_{\mu}-T_{N_{1}^{\prime}}^{\infty}-T_{N_{2}^{\prime}}^{\infty}
$$

and compared to the $1 \mathrm{p} 1 \mathrm{~h} E_{m}^{I A}$ values discussed in the previous section. The excitation of two nucleons leads to a bigger offset of the $E_{m}^{I A}$ values and a longer energy tail compared to that of the $1 \mathrm{p} 1 \mathrm{~h}$ distribution. The immediate consequence of this implementation 
is that the average $E_{m}^{I A}$ is around $45 \mathrm{MeV}$ which is larger than the one of the $\mathrm{CC} 1 \mathrm{p} 1 \mathrm{~h}$ and larger than previous implementations of $\mathrm{CC} 2 \mathrm{p} 2 \mathrm{~h}$ models, where the typical $\mathrm{CC} 1 \mathrm{p} 1 \mathrm{~h}$ $E_{m}^{I A}$ were implemented. This correction will reduce the overall $2 \mathrm{p} 2 \mathrm{~h}$ cross-section for low energy transfers.

The remaining neutrino-nucleus interaction channels, mostly with associated pion production, are simulated based on the existing NEUT [8] Monte Carlo event generator. The resonant pion production is based on the Rein-Sehgal model [22], taking into account eighteen resonances with masses below $2 \mathrm{GeV}$ and their interference terms, with the axial

mass fixed to $M_{\mathrm{A}}^{\mathrm{RES}}=1.21 \mathrm{GeV}$. This model has been compared to experimental data by the T2K collaboration showing remarkable agreement [14]. Neutral current and charged current coherent pion production is simulated using the Rein-Sehgal model in ref. [23]. The CC coherent pion production includes PCAC (partially conserved axial-vector current) and lepton mass corrections, as discussed in [24]. DIS processes are simulated using the GRV98 [25] parton distribution, with low- $\mathrm{Q}^{2}$ corrections from the Bodek and Yang model [26]. Secondary interactions of pions inside the nucleus are simulated using an intranuclear cascade model based on the method developed by Salcedo et al. [27], tuned to external $\pi-{ }^{12} \mathrm{C}$ data [28].

\section{Comparison to experimental data}

In this section we discuss the comparison of the predictions from the model with recent data from MINERvA and T2K cross sections with no pions in the final state. The implementation of the model inside NEUT allows us to make a direct comparison with the experimental cross-sections since all the interaction channels are considered including, the transport of the nucleons and pions inside of the nucleus. The data selected include inclusive muon kinematics and TKI variables to explore the limits of the hadron kinematic predictions of the model.

\subsection{Event simulation and data selection}

Events are simulated using the NEUT package with the CCQE and CC2p2h reactionmechanisms described above. We take the fluxes from the experiment releases according to their best understanding. The simulation is done for three configurations of the nucleon rescattering probability (NrSP): nominal, and $50 \%$ and $150 \%$ strengths. This is only applied to the proton re-scattering while pions are kept to their nominal NEUT values. We select events according to the particles emitted by the nucleus after the interaction taking into account the event acceptance of the experiments as described in their published designs.

\subsubsection{T2K data samples}

The neutrino T2K data-sample has different selections obtained from the off-axis muon neutrino beam, which peaks around $0.6 \mathrm{GeV}$ but it contains a large energy-tail ranging to the region of tens of $\mathrm{GeV}$. The $\mathrm{CC}$ inclusive measurement considers only the muon production kinematics ignoring all hadronic activity [12]. The T2K selection criterion for $\mathrm{CC} 0 \pi[13]$ requires no charged or neutral pions in the final state. Based on this selection, 
T2K provides double differential cross-sections for carbon and oxygen nuclear targets. The comparison is performed only on carbon data to keep a common nuclear target across the different measurements and experiments.

The T2K selection criterion for the single TKI variables analysis of the $\mathrm{CC} 0 \pi 1 p$ sample requires the detection of a muon and proton with the following conditions [21]:

- $0.45 \mathrm{GeV}<\left|\vec{p}_{p}\right|<1.0 \mathrm{GeV}$.

- $\cos \theta_{p}>0.4$

- $0.25 \mathrm{GeV}<\left|\vec{p}_{\mu}\right|<10 \mathrm{GeV}$.

- $\cos \theta_{\mu}>-0.6$

with $\theta_{p}$ the polar angle of the outgoing proton, which has a modulus of the three-momentum $\left|\vec{p}_{p}\right|$. T2K presents also a slightly less restrictive selection criterion for $\mathrm{CC} 0 \pi 1 p$ requiring the detection of a muon and proton with the following conditions [21]:

- $0.45 \mathrm{GeV}<\left|\vec{p}_{p}\right|<1.0 \mathrm{GeV}$.

- $\cos \theta_{p}>0.4$

\subsubsection{MINERvA data samples}

MINERvA, with an average neutrino energy of $3.5 \mathrm{GeV}$, published different event selections: the $\mathrm{CC}$ inclusive, the $\mathrm{CC} 0 \pi$ that removes events with detected charged $\pi$ 's and electromagnetic activity to eliminate events with $\pi^{0}$, and the $\mathrm{CC} 0 \pi 1 p$ sample which is a sub-sample of $\mathrm{CC} 0 \pi$ requesting the presence of an identified proton in the final state. Another data sample from MINERvA, the so-called available energy [29], requires full simulation of the detector simulation that falls beyond the capabilities of this work.

The CC inclusive selection in MINERvA requires [16] a muon with polar angle $\left(\theta_{\mu}\right)$ below $20^{\circ}$. The selection criteria for $\mathrm{CC} 0 \pi$ demands in addition [17]:

- a muon with momentum $\left|\vec{p}_{\mu}\right|$ between $1.5 \mathrm{GeV}$ and $10 \mathrm{GeV}$,

- neither charged, nor neutral pions escaping the nucleus.

- no $\gamma$ with energies about $10 \mathrm{MeV}$. We check this cut actually do not affect the MC prediction for CCQE and CC2p2h.

The selection criteria for $\mathrm{CC} 0 \pi 1 p$ requires in addition $[15,19,20]$ :

- a proton with polar angle $\left(\theta_{p}\right)$ below $70^{\circ}$.

- a proton with momentum between $0.45 \mathrm{GeV}$ and $1.2 \mathrm{GeV}$.

These two conditions are applied to protons leaving the nucleus after the nucleon re-scattering. 


\begin{tabular}{|c|c|c|c|c|c|c|}
\hline Component & $\mathrm{CH}$ & $\mathrm{O}$ & $\mathrm{Al}$ & $\mathrm{Si}$ & $\mathrm{Cl}$ & $\mathrm{Ti}$ \\
\hline Weight (\%) & 95.02 & 3.18 & 0.26 & 0.27 & 0.55 & 0.69 \\
Nuclei (\%) & 96.71 & 2.63 & 0.13 & 0.13 & 0.21 & 0.19 \\
\hline Nucleons (\%) & 95.04 & 3.18 & 0.26 & 0.27 & 0.55 & 0.69 \\
\hline
\end{tabular}

Table 1. MINERvA target nuclear composition in weight fraction and the translation to the fractional composition in nuclei. Last row shows the fraction of nucleons of each of the spices with respect to the total. Data taken from ref. [30].

\begin{tabular}{|c|c|c|c|c|}
\hline Component & $\mathrm{CH}$ & $\mathrm{O}$ & $\mathrm{Si}$ & $\mathrm{Ti}$ \\
\hline Weight (\%) & 95.02 & 3.18 & 0.27 & 0.69 \\
Nuclei (\%) & 97.04 & 2.64 & 0.12 & 0.19 \\
\hline Nucleons (\%) & 95.82 & 3.21 & 0.27 & 0.70 \\
\hline
\end{tabular}

Table 2. The same as table 1, but for the T2K experiment. Data taken from ref. [31].

\subsection{Target composition}

MINERvA and T2K targets are composed materials made of several components: $\mathrm{CH}$, $\mathrm{O}, \mathrm{Al}, \ldots$. Proportions in weight and nuclear content are given in tables 1 and 2. To simulate the experimental composition we take the approximation of selecting only the main 3 components: $\mathrm{C}, \mathrm{H}$ and $\mathrm{O}$ in proportions given in the tables. This actually has an appreciable effect on the selection mainly because of the large Fermi momentum and nuclear radius of the oxygen affecting the nucleon transport in the nucleus, accounting for secondary collisions. Both experiments have the same target material (plastic scintillators) with very similar composition. The correction to the cross-section prediction introduced by be oxygen contribution is estimated by our models to be at the order of a percent. The exception to this treatment is the $\mathrm{CC} 0 \pi$ cross-section that is reported by $\mathrm{T} 2 \mathrm{~K}$ for a pure carbon target [13] and not in hydrocarbon as for the CC inclusive and MINERvA.

\subsection{Inclusive cross-sections}

In this section we compare our model predictions with the $\mathrm{CC}$ inclusive and $\mathrm{CC} 0 \pi$ measurements. The selection of events in the first reaction relies on the muon kinematics and ignore the hadronic component of the interaction. This sample allows to make a datato-model comparison with a reduced bias from selection and detector acceptances, but it relies on the proper description of other interaction channels in the Monte Carlo. The $\mathrm{CC} 0 \pi$ sample requires that there are no pions observed in the final state. This selection, reduces the contribution of channels beyond $\mathrm{CC} 1 \mathrm{p} 1 \mathrm{~h}$ and $\mathrm{CC} 2 \mathrm{p} 2 \mathrm{~h}$ but it is affected by the correct modelling of both, the pion re-interactions inside the nucleus and the primary pion production mechanism.

T2K published both the $\mathrm{CC}$ inclusive [12] and the $\mathrm{CC} 0 \pi$ [13] cross-sections as a double differential distributions as function of the muon momentum and angle. The comparisons of these data-samples and the NEUT model predictions are shown in the left and right panels 

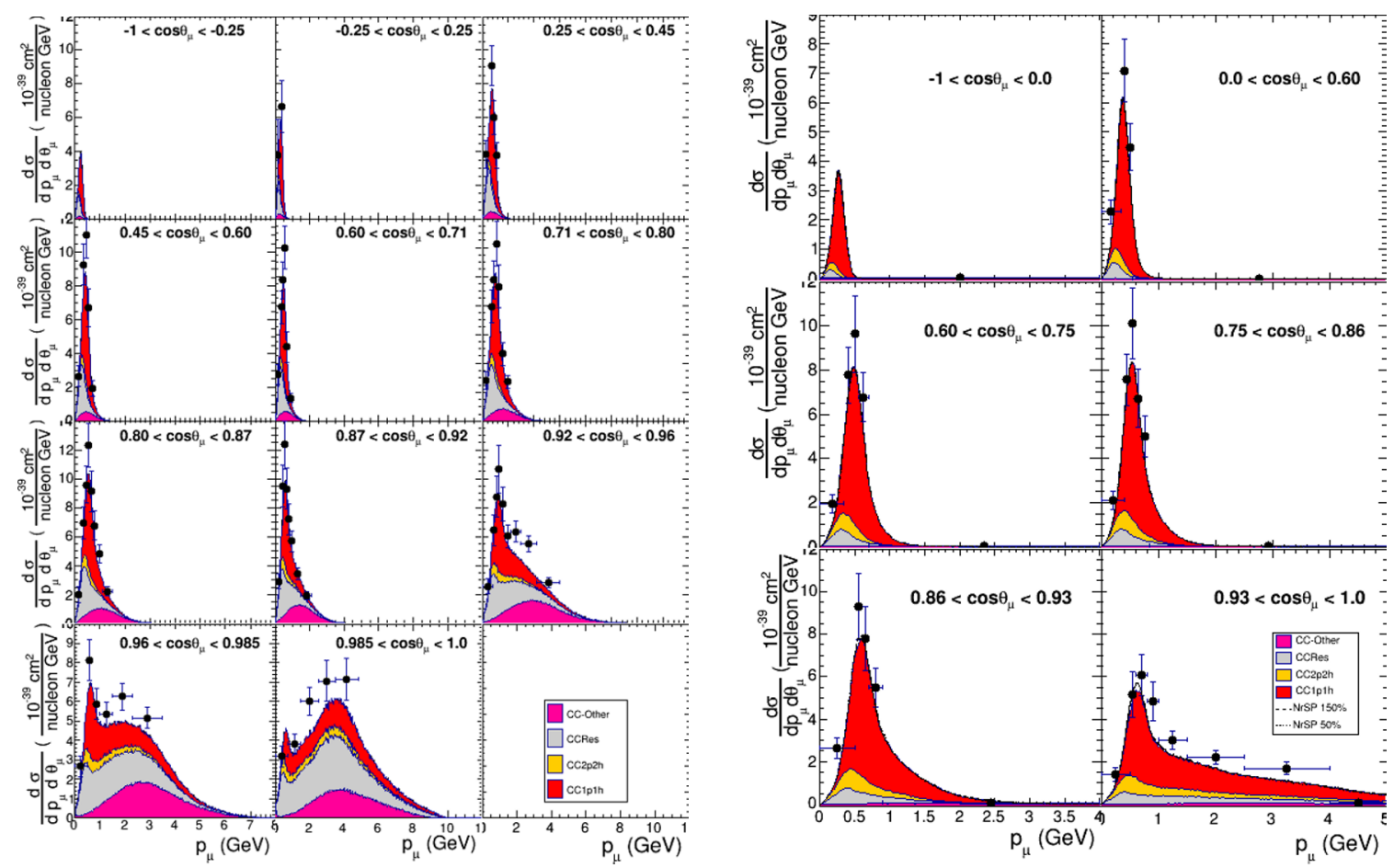

Figure 5. T2K CC (left) and $\mathrm{CC} 0 \pi$ (right) inclusive double differential cross-section $d \sigma / d p_{\mu} d \cos \theta_{\mu}$. Data, taken from refs. [12] and [13], respectively, are compared to the results obtained from the present implementation of NEUT with the numerical values for the $\chi^{2}$-likelihood test compiled in table 3. Last bin accumulates all the statistics until the kinematic limit of $30 \mathrm{GeV}$.

of figure 5, respectively. The $\mathrm{CC}$ inclusive spectrum shows a sizeable contribution from resonant and deep inelastic scattering (DIS) channels, while for the $\mathrm{CC} 0 \pi$ such components are considerably reduced, see also table 6 . The remaining CCRes contribution comes from pion absorption in the nucleus. The model predicts reasonably well the tendencies in the experimental data.

MINERvA published the muon 2D longitudinal and transverse momentum distributions for the CC inclusive cross-section measurements [16] (see top panels of figure 6). The cross-section is dominated by DIS, but there are regions $\left(1.5 \mathrm{GeV}<\mathrm{p}_{\|}<5.0 \mathrm{GeV}\right)$ where the $\mathrm{QE}$ and $2 \mathrm{p} 2 \mathrm{~h}$ contributions are relevant. The agreement with the present version of NEUT in the regions where CCQE is relevant is good, while the high longitudinal momentum histograms show cross-section predictions lower than data. The $\mathrm{CC} 0 \pi$ sample from MINERvA is also shown in figure 6 (bottom panels). It can be observed that the predictions are qualitatively similar to those found for the MINERvA CC inclusive case, lower than the data for large $\left(p_{\|}>5.0 \mathrm{GeV}\right)$, but also for low $\left(p_{\|}<3.5 \mathrm{GeV}\right)$ longitudinal momenta.

To estimate the agreement data-model, we computed the $\chi^{2}$-merit function between the model predictions and data using the full error covariance matrices provided by the T2K and MINERvA experiments. The results are compiled in tables 3 and 4, respectively. The comparison is done for three different scale factors of the proton-nucleon cross-sections accounting for secondary collisions. Absolute $\chi^{2}$-values for the T2K CC-inclusive reaction 

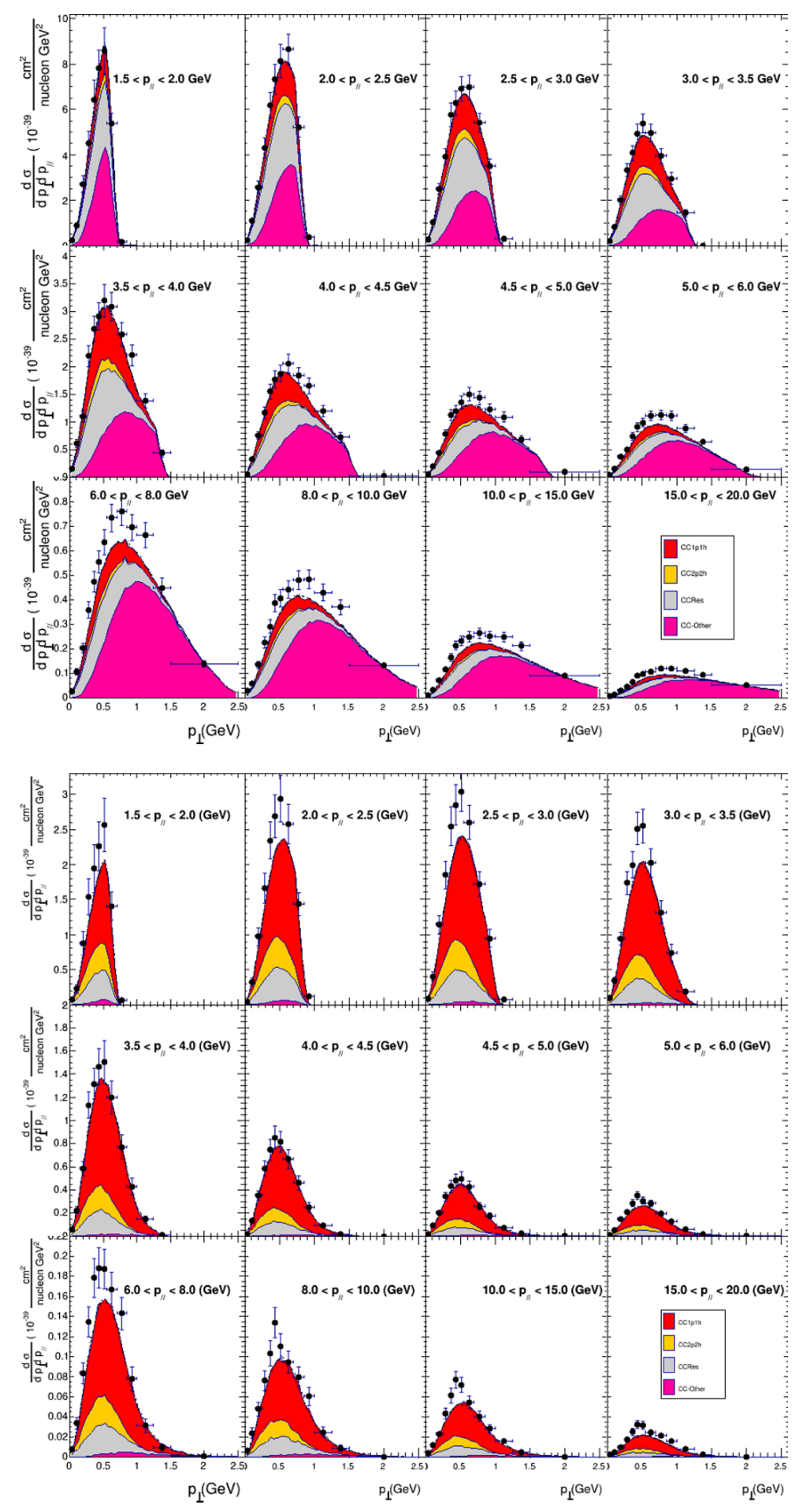

Figure 6. MINERvA CC (top) and $\mathrm{CC} 0 \pi$ (bottom) inclusive double differential cross-section $d^{2} \sigma / d p_{\perp} d p_{\|}$. Data, taken from refs. [16] and [17], respectively, are compared to the results obtained from the present implementation of NEUT, with the $\chi^{2}$-likelihood tests compiled in table 4 . 


\begin{tabular}{|c|c|c|c|c|}
\hline variable & dof & nominal & NrSP: $50 \%$ & NrSP: $150 \%$ \\
\hline CC inclusive & 71 & 110 & 104 & 104 \\
CC0 $\pi$ inclusive & 29 & 17 & 17 & 17 \\
\hline$\delta \alpha_{T}$ & 8 & 35 & 29 & 43 \\
$\delta \phi_{T}$ & 8 & 19 & 27 & 36 \\
$\delta p_{T}$ & 8 & 7 & 32 & 13 \\
$|\Delta \vec{p}|$ & 49 & 261 & 193 & 398 \\
$\Delta \theta$ & 35 & 1021 & 798 & 1322 \\
$\Delta|\vec{p}|$ & 49 & 130 & 111 & 177 \\
\hline
\end{tabular}

Table 3. $\chi^{2}$-likelihood test for T2K variables. The number of degrees of freedom (dof) is given in the second column, while in the another three ones, results obtained for different distributions and three $\mathrm{NrSP}$ — proton re-scattering probability — configurations (nominal, 50\% and $150 \%$ strengths) are collected.

are close to the dof and they show very little variations between the three examined scenarios. This is because re-scattering effects should not affect the inclusive cross section, and the observed differences should be produced by MC fluctuations (note that the three simulations are statistically independent). The $\chi^{2}$-value found in this work is almost a factor of two smaller than those presented in the experimental paper (NEUT 5.3.2 and GENIE [32] 2.8.0). The theoretical description of the T2K CC0 $\pi$ data sample is better than that achieved for the inclusive one, with $\chi^{2}$-value around 17 for 29 degrees of freedom. In this case, the different NrSP assumptions do not practically alter the results, as expected since the kinematics of the proton is not used in the $\mathrm{CC} 0 \pi$ event selection. The $\chi^{2}$ value achieved with the present scheme is among the best reported in the T2K paper and it approaches the model "NEUT 5.4.1 LFG" in table V of [13], since both largely share the physics implementation.

On the other hand, the best-fit $\chi^{2}$ values for the CC inclusive MINERvA data sample, collected in table 4 , are among the best five reported in the experimental paper. Since this is dominated by the DIS cross-section, as discussed in figure 6, this result also confronts the prediction for this reaction channel in NEUT. The description of the MINERvA $\mathrm{CC} 0 \pi$ events, where DIS has been practically removed, significantly improves, and it is quantitatively slightly poorer than that seen above for T2K. Nevertheless, the $\chi^{2}$ value is among the best two reported in the experimental paper and significantly better than any of the models including $2 \mathrm{p} 2 \mathrm{~h}$ contributions. NEUT predictions for MINERvA CC0 $\pi$ show apparently a larger, though still soft, dependence on the proton re-scattering probability.

\subsection{Transverse kinematic-imbalance (TKI) variables}

TKI variables $[33,34]$, depicted in figure 7 , lead to observable distributions with minimal dependence on neutrino energy, which provide direct constraints on nuclear effects in (anti)neutrino-nucleus interactions. Obviously, the measurement of the distribution of 


\begin{tabular}{|c|c|c|c|c|}
\hline variable & dof & nominal & NrSP: $50 \%$ & NrSP: $150 \%$ \\
\hline CC inclusive & 144 & 420 & 435 & 420 \\
CC0 $\pi$ & 144 & 208 & 247 & 213 \\
\hline$\delta \alpha_{T}$ & 12 & 21 & 25 & 18 \\
$\delta \phi_{T}$ & 23 & 47 & 105 & 46 \\
$\delta p_{T}$ & 24 & 89 & 155 & 56 \\
$\delta p_{T_{x}}$ & 32 & 63 & 106 & 43 \\
$\delta p_{T_{y}}$ & 33 & 56 & 108 & 40 \\
$\left|\vec{p}_{\mu}\right|$ & 32 & 29 & 41 & 26 \\
$\theta_{\mu}$ & 19 & 23 & 24 & 22 \\
$p_{p}$ & 25 & 30 & 33 & 31 \\
$\theta_{p}$ & 26 & 49 & 62 & 43 \\
$p_{n}$ & 24 & 107 & 202 & 86 \\
\hline
\end{tabular}

Table 4. Same as in table 3, but for MINERvA experiment.

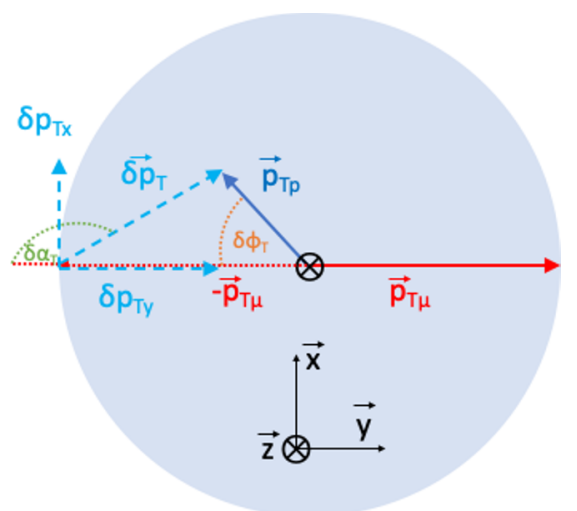

Figure 7. Angular $\left(\delta \alpha_{T}, \delta \phi_{t}\right)$ and transverse momentum variables $\left(\vec{\delta} p_{T}, \delta p_{T_{X}}, \delta p_{T_{Y}}\right)$ in a reference system, where the neutrino is entering perpendicular to the plane.

these variables require the observation of the outgoing proton (neutrino reactions) or the neutron (anti-neutrino reactions) in the final state. The missing transverse, with respect to the neutrino direction, momentum is computed as

$$
\left|\delta \vec{p}_{T}\right| \equiv\left|\vec{p}_{T}\right|=\frac{\left|\left(\vec{p}_{\mu}+\vec{p}_{p}\right) \times \vec{p}_{\nu}\right|}{\left|\vec{p}_{\nu}\right|}
$$

In addition, the missing transverse momentum is separated in two components in relation to the $\nu-\mu$ reaction plane, defined by the neutrino and the emitted charged lepton. One of the transverse components is contained in the reaction plane $\delta p_{T_{y}}$, while the another one, $\delta p_{T_{x}}$, is perpendicular to this plane [19]. 
On the other hand, the TKI angular variables read:

$$
\begin{aligned}
& \delta \phi_{T}=\arccos \left[\frac{-\vec{p}_{T_{p}} \cdot \vec{p}_{T_{\mu}}}{\left|\vec{p}_{T_{p}}\right|\left|\vec{p}_{T_{\mu}}\right|}\right] \\
& \delta \alpha_{T}=\arccos \left[\frac{-\vec{p}_{T} \cdot \vec{p}_{T_{\mu}}}{\left.\mid \overrightarrow{p_{T}|\cdot| \vec{p}_{T_{\mu}} \mid}\right]}\right.
\end{aligned}
$$

where $\vec{p}_{T}=\vec{p}_{T_{\mu}}+\vec{p}_{T_{p}}$, with $\vec{p}_{T_{\mu}}$ (or $\vec{p}_{\perp}$, as used in figure 6 ) and $\vec{p}_{T_{p}}$ the transverse projections of the muon and proton momenta to the neutrino direction. This discussion focuses on the QE-like process $\nu_{\mu}+A \rightarrow \mu^{-}+p+X$, where $X$ is a final-state hadronic system consisting of the nuclear remnant with possible additional protons but without pions that indicate resonant or other processes. There is an imbalance, $\delta \vec{p}$, between the initial neutrino momentum and the sum of final-state lepton and hadron momenta as a result of nuclear effects. Under the assumption that $X$ is just the remnant nucleus, with $(A-1)$ nucleons, then $|\delta \vec{p}|$ gives the magnitude of its recoil momentum, which can be obtained [35] independently of the unknown incident neutrino energy. Moreover, assuming perfect balance of momentum in the reaction (see discussion of eqs. (2.2) and (2.3)), $|\delta \vec{p}|$ can be identified to the neutron target momentum, which is then given in terms of measurable quantities [15, 35]

$$
\left|\vec{p}_{n}\right|=\sqrt{\left|\vec{p}_{T}\right|^{2}+\left|\vec{p}_{L}\right|^{2}}
$$

with

$$
\left|\vec{p}_{L}\right|=\frac{\left(M_{A}-E_{\mu}-E_{p}^{\infty}+\left|\vec{p}_{L_{p}}\right|\right)^{2}-\left(M_{A-1}^{\prime}\right)^{2}-\left|\vec{p}_{T}\right|^{2}}{2\left(M_{A}-E_{\mu}-E_{p}^{\infty}+\left|\vec{p}_{L_{p}}\right|+\left|\vec{p}_{L_{\mu}}\right|\right)}
$$

where $\vec{p}_{L_{p}}$ and $\vec{p}_{L_{\mu}}$ (or $\vec{p}_{\|}$, as used in figure 6) denote the projections of the corresponding three-vectors on the direction of the incoming neutrino. For the MINERvA measurement of refs. $[15,19,20], M_{A}$ is the mass of the carbon target, while for $M_{A-1}^{\prime}$ is taken $\left(M_{A}-\right.$ $m_{n}+E_{b}$ ), with $E_{b}$ the nucleon binding energy that is fixed to $27.13 \mathrm{MeV}$ and $m_{n}$ is the mass of the neutron. ${ }^{7}$

The T2K collaboration considered additional distributions based on the neutrino energy reconstruction formula used in neutrino oscillation experiments. This prescription assumes a genuine QE event where the target nucleon is at rest, and the neutrino energy is reconstructed assuming energy and momentum conservation (see for instance ref. [4]):

$$
E_{\nu}^{\mathrm{rec}}=\frac{m_{p}^{2}-m_{\mu}^{2}+2 E_{\mu}\left(m_{n}-E_{b}\right)-\left(m_{n}-E_{b}\right)^{2}}{2\left(\left(m_{n}-E_{b}\right)-E_{\mu}+\left|\vec{p}_{\mu}\right| \cos \theta_{\mu}\right)}
$$

where $\theta_{\mu}$ is the angle of the muon with respect to the average neutrino direction, $m_{\mu}$ is the mass of the muon, $m_{p}$ is the mass of the outgoing proton and the constant $E_{b}$ is fixed to $25 \mathrm{MeV}$ in the experimental $\mathrm{T} 2 \mathrm{~K}$ results [21]. Once the neutrino energy is known, one can compute the so-called inferred proton momentum:

$$
\vec{p}_{p}^{\text {inf }}=\vec{p}_{\nu}^{\text {rec }}-\vec{p}_{\mu}
$$

\footnotetext{
${ }^{7}$ Note, this is the prescription used in the experimental work, which should be used to compare to the event-distributions provided in that paper, but it does not correspond to the energy-balance of eq. (2.7) proposed here for genuine QE processes.
} 
New observables are build by comparing the inferred momentum with the experimentally measured proton momentum $\left(\vec{p}_{p}^{\infty}\right)$ :

$$
\begin{aligned}
|\Delta \vec{p}| & =\left|\vec{p}_{p}^{\inf }-\vec{p}_{p}^{\infty}\right| \\
\Delta|\vec{p}| & =\left|\vec{p}_{p}^{\inf }\right|-\left|\vec{p}_{p}^{\infty}\right| \\
\Delta \theta & =\arccos \left[\frac{\vec{p}_{p}^{\infty} \cdot \vec{p}_{\nu}}{\left|\vec{p}_{p}^{\infty}\right|\left|\vec{p}_{\nu}\right|}\right]-\arccos \left[\frac{\vec{p}_{p}^{\inf } \cdot \vec{p}_{\nu}}{\left|\vec{p}_{p}^{\inf }\right|\left|\vec{p}_{\nu}\right|}\right]
\end{aligned}
$$

The MINERvA Collaboration reported different cross-sections from its $\mathrm{CC} 0 \pi 1 p$ data sample $[15,19,20]$ :

- Inclusive lepton momentum and angle.

- Reconstructed kinematics such as the target nucleon momentum $\left|\vec{p}_{n}\right|$.

- Visible proton momentum.

- TKI angles: $\delta \phi_{T}$ and $\delta \alpha_{T}$.

- Transverse momentum balance: $\delta p_{T}, \delta p_{T_{x}}, \delta p_{T_{y}}$.

while T2K reported on slightly different set of variables from its $\mathrm{CC} 0 \pi 1 p$ sample [21]:

- Transverse angles (TKI variables with first selection criterion): $\delta \phi_{T}$ and $\delta \alpha_{T}$, and the missing transverse momentum $\left|\delta \vec{p}_{T}\right|$

- Proton momentum balance (Inferred variables with the second selection criterion): $|\Delta \vec{p}|, \Delta \theta$ and $\Delta|\vec{p}|$ in bins of muon momentum and angle.

Experimental and NEUT predictions for the TKI angular variables $\delta \alpha_{T}$ and $\delta \phi_{T}$, and the missing transverse momentum $\left|\delta \vec{p}_{T}\right|$, both for MINERvA (left) and T2K (right) are shown in figures 8, 9 and 10, respectively. Absolute $\chi^{2}$-values obtained from the comparison with data are compiled in tables 3 and 4 , respectively. The NEUT results for the TKI variables are in general in an acceptable agreement with both MINERvA and T2K data, though the MINERvA distributions are better described. As expected, we observe some significant dependence on the proton re-scattering probability, which is reflected in the $\chi^{2}$-likelihood tests. We should point out that NrSP effects change not only the overall normalization, but also the shape of the distributions. This is clearly visible, for instance, in the $\mathrm{CC} 0 \pi 1 p$ T2K [21] $\delta \alpha_{T}$ distributions depicted in figure 8. There, we see that re-scattering effects become more important in the region of smallest $\delta \alpha_{T}$ angles. This observation is in order for some other distributions discussed below in this subsection.

The transverse momentum components contain different information. The component $\delta p_{T_{x}}$ is expected to be symmetric around zero, with a width which depends on the target neutron momentum and further re-scattering effects, while $\delta p_{T_{y}}$ can be asymmetric due to leading effect of the neutrino boost. Results are shown in figure 11. The tendency is very well described by the model with the long tails dominated by $2 \mathrm{p} 2 \mathrm{~h}$, resonant and, DIS mechanisms in the $\delta p_{T_{x}}\left(\delta p_{T_{y}}\right)$ distribution. The $\chi^{2}$ comparison, see table 4 shows an 

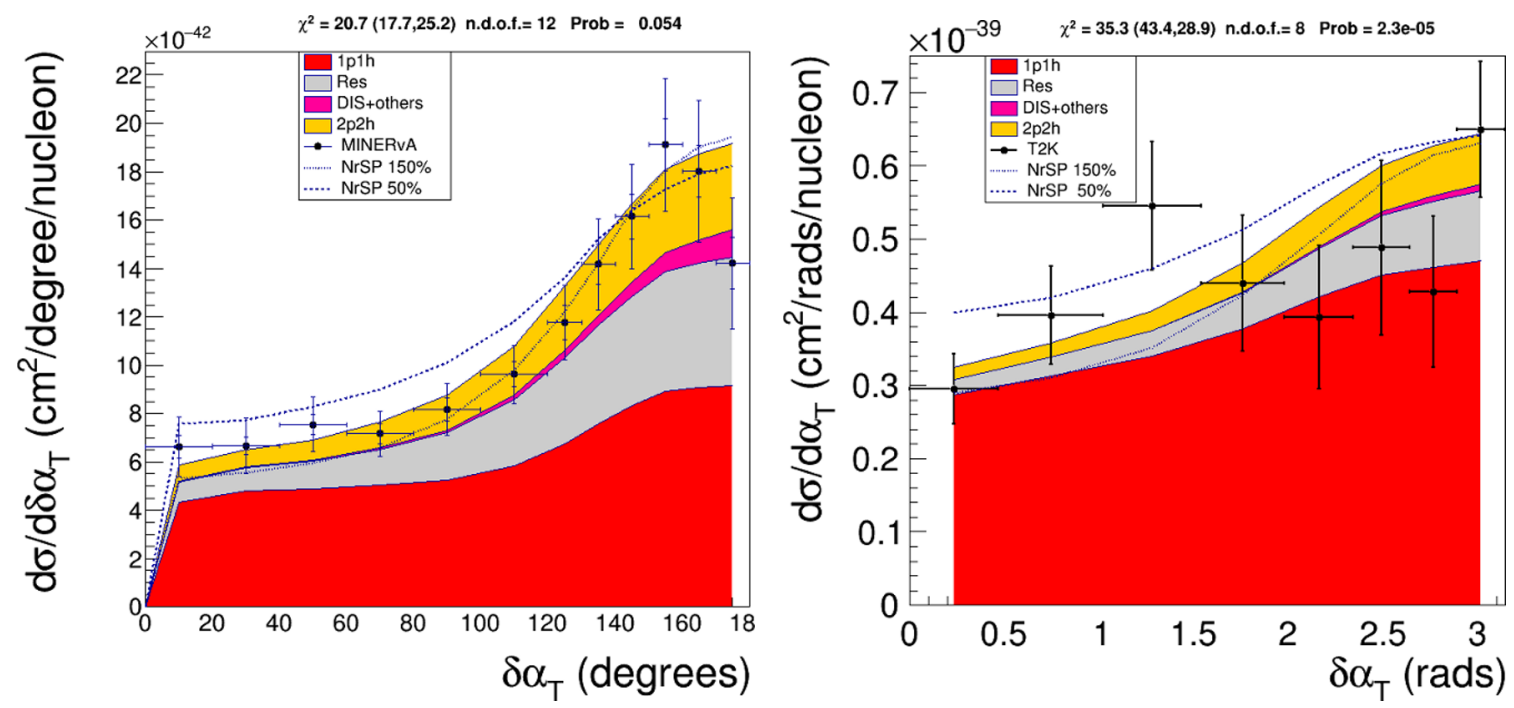

Figure 8. CC0 $\pi 1 p$ MINERvA $[15,19,20]$ (left) and T2K [21] (right) $\delta \alpha_{T}$ distributions compared to the predictions of the implementation of NEUT in this work. The simulation has been done for three configurations of the NrSP (nominal, $50 \%$ and $150 \%$ strengths) and the obtained absolute $\chi^{2}$-values are compiled in tables 3 and 4 .
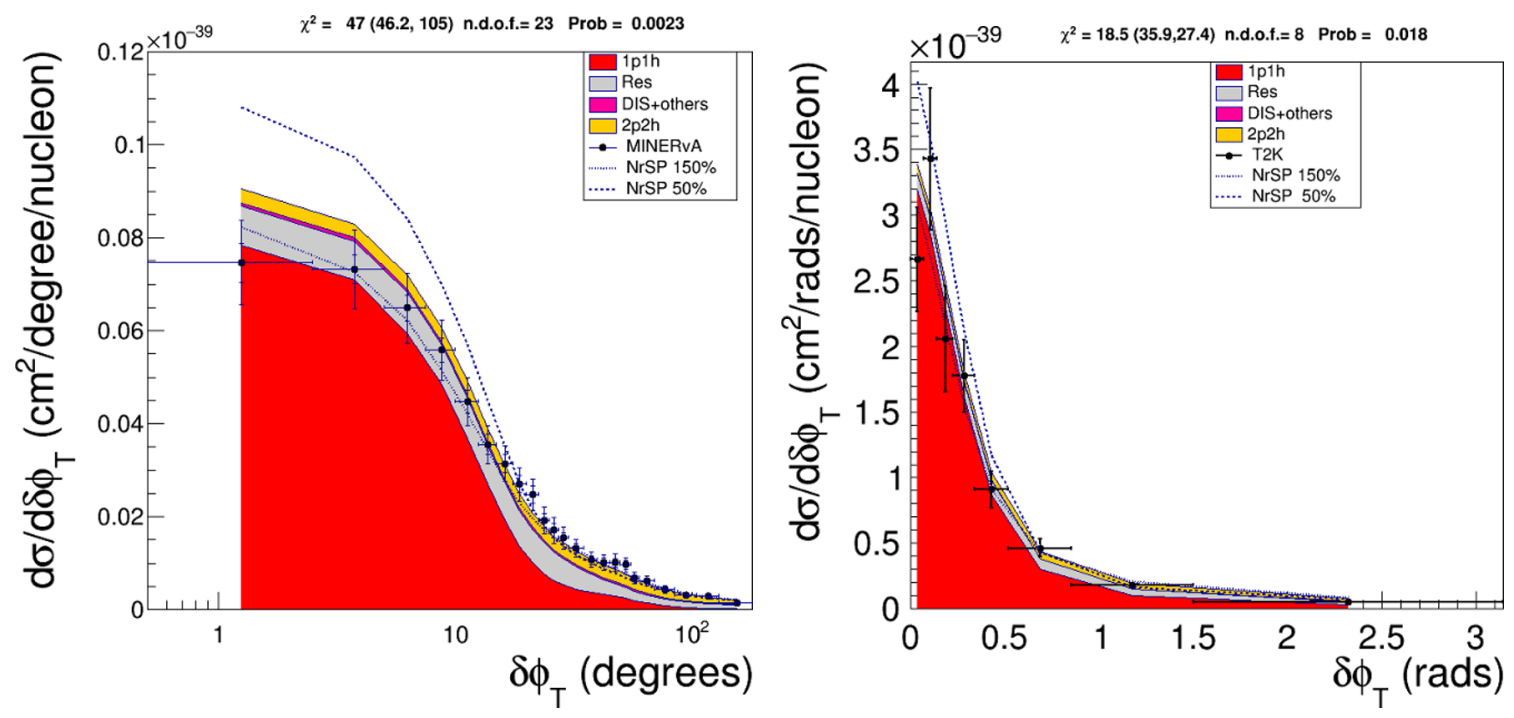

Figure 9. CC0 $\pi 1 p$ MINERvA $[15,19,20]$ (left) and T2K [21] (right) distributions for the TKI angular variable $\delta \phi_{T}$. Details of the comparison with NEUT results as in figure 8 .

excellent agreement with a preference for an increase in the NrSP, which reduces the contribution of the CCQE channel by reducing the probability of proton tagging in detectors. This tendency is shared by most of the other TKI observables.

MINERvA collaboration also reported $\left|\vec{p}_{\mu}\right|$ and $\theta_{\mu}$ distributions from its CC $0 \pi 1 p$ MINERvA sample $[15,19,20]$. The comparison of these data with the current implementation of NEUT is shown in figure 12. We find a quite good description of these two event distributions, with $\chi^{2} / d o f$ around one (see table 4) for nominal NrSP, and some dependence on this latter input as expected when analysing $\mathrm{CC} 0 \pi 1 p$ data-samples. 

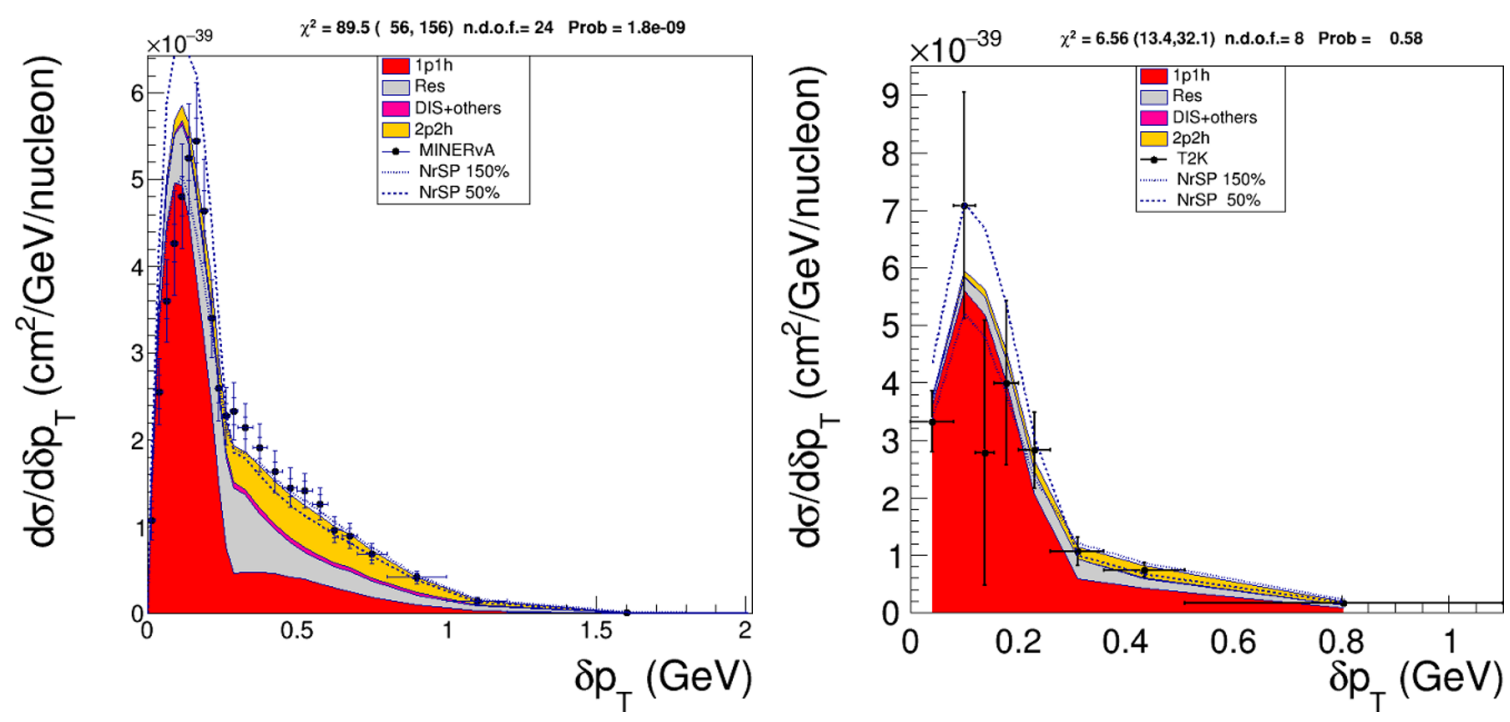

Figure 10. CC0 $\pi 1 p$ MINERvA $[15,19,20]$ (left) and T2K [21] (right) distributions for the missing transverse momentum $\left|\delta \vec{p}_{T}\right|$. Details of the comparison with NEUT results as in figure 8.

The experimental results with a visible proton in the final state are biased towards high momentum transfers since the proton should have at least $450 \mathrm{MeV}$ to be detected. The typical proton momentum and angle distributions in MINERvA are shown in figure 13. On the contrary, the samples with additional invisible protons do not suffer from large momentum transfer biases. The difference between the two are dominated by low $|\vec{q}|$ contributions with feed-down background cause by NrSP. These tendencies can be observed in figures 25 and 26 of appendix A, where the model estimation for the energy $\left(q^{0}=E_{\nu}-E_{\mu}\right)$ and momentum $\left(|\vec{q}|=\left|\vec{p}_{\nu}-\vec{p}_{\mu}\right|\right)$ transfer distributions for the MINERvA and T2K CC inclusive, $\mathrm{CC} 0 \pi$ and $\mathrm{CC} 0 \pi 1 \mathrm{p}$ event selections are shown.

The overall agreement with NEUT is good, showing the importance of $2 \mathrm{p} 2 \mathrm{~h}$ mechanisms. The results for the TKI variables are good for the MINERvA data, with statistically acceptable values of $\chi^{2} / d o f$ for most of the cases. The worst comparison is obtained for the reconstructed $\left|\vec{p}_{n}\right|$ variable, where a large discrepancy is observed in the region around $0.3 \mathrm{GeV}$ (see figure 14). This is at the transition from the CC1p1h dominated cross-section to the one dominated by resonance and $\mathrm{CC} 2 \mathrm{p} 2 \mathrm{~h}$ mechanisms. This is actually the most distinctive difference in all the comparisons of this work and a nice reference observable to try model variations. As it is shown in figure 14 and table 4, the variation of the proton re-scattering probability does no alleviate the discrepancy. Since, the high momentum $\left(\left|\vec{p}_{n}\right| \geq 0.5 \mathrm{GeV}\right)$ region is well reproduced by the model, the discrepancy seems to be led by the transition, either from non described tails in the CC1p1h, which might come from high energy neutron target components predicted by realistic SFs, or by a miss-representation of resonant or $\mathrm{CC} 2 \mathrm{p} 2 \mathrm{~h}$ models. In any case, it seems that a re-weight of the cross-section will not improve the agreement.

The agreement with $\mathrm{T} 2 \mathrm{~K} \mathrm{CC} 0 \pi 1 \mathrm{p}$ data is less impressive and the obtained $\chi^{2} / d o f$, see table 3, are large for all the three observables $|\Delta \vec{p}|,|\Delta \theta|$ and $\Delta|p|$ reported in [21], and 

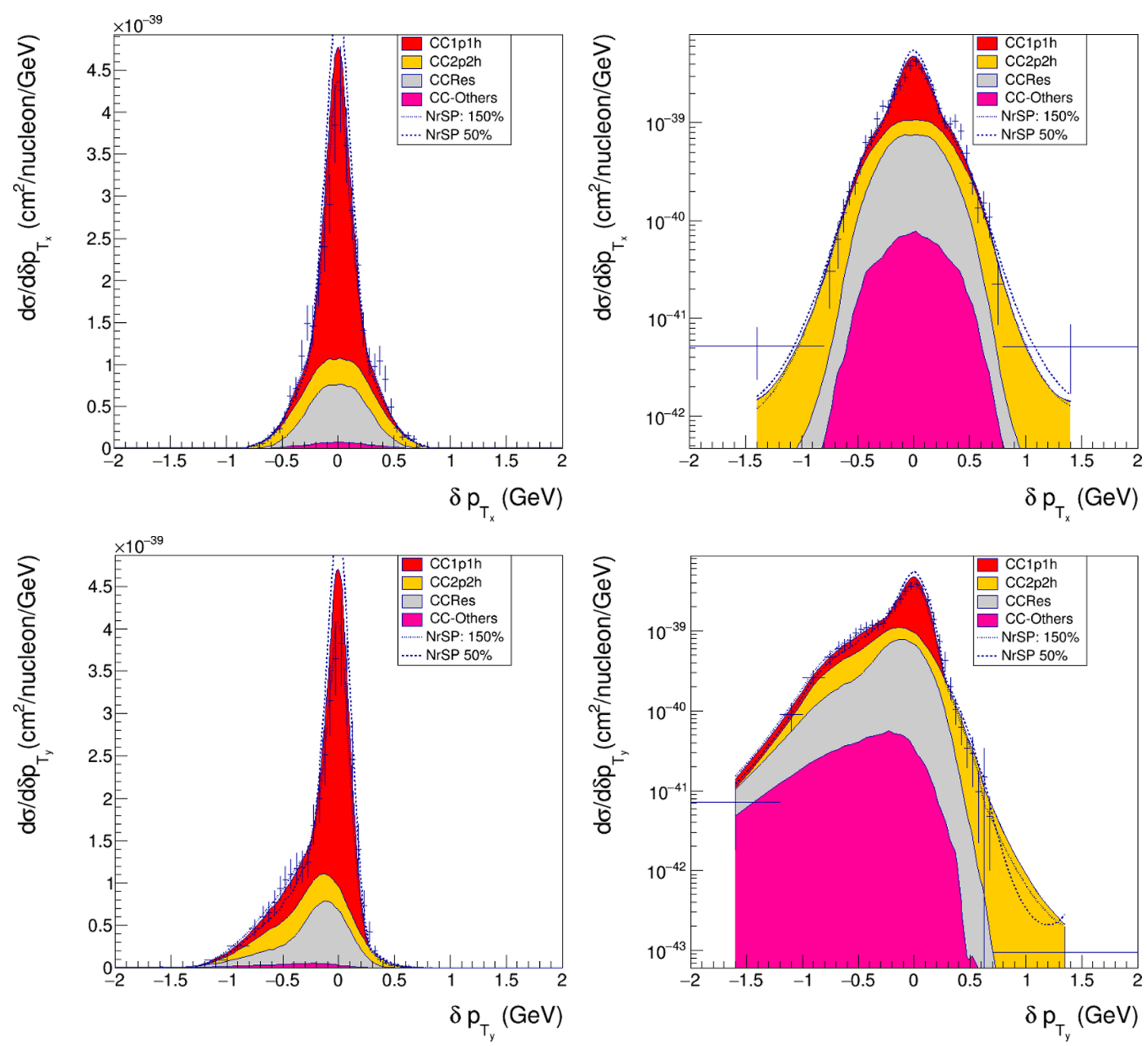

Figure 11. MINERvA $\delta p_{T_{x}}$ (top) and $\delta p_{T_{y}}$ (bottom) distributions $[15,19,20]$. Left and right panels show the comparison (details as in figure 8) with NEUT in linear and logarithmic scales, respectively.

shown here in figures $15-17$. The worst situation is found for the $\Delta \theta$ distribution, with the largest contributions to $\chi^{2}$ produced by the negative $\Delta \theta$ bins. There, the number of events is always very small independent of the muon-kinematics, and the present model fails to properly describe those data, though one should bear in mind that these bins have a negligible weight in the totally integrated cross section. Indeed, if these bins are removed, the merit-function $\chi^{2}$ is reduced to 66 for 30 degrees of freedom. The agreement is slightly better for the re-scaling factor $\mathrm{NrSP}=0.5$ that reduces the scattering of the outgoing proton in the nucleus (54 for negative values of $\Delta \theta$ ). The $\chi^{2}$ figures for $|\Delta \vec{p}|$, and $\Delta|p|$ are also reduced with the re-scaling factor $\mathrm{NrSP}=0.5$.

In summary from the results of tables 3 and 4, we conclude that MINERvA TKI distributions are better described with the $150 \%$ enhanced NrSP, while the T2K ones favor either nominal $\left(\delta \phi_{T}\right.$ and $\left.\delta p_{T}\right)$ or the $50 \%$ reduced $\operatorname{NrSP}\left(\delta \alpha_{T},|\Delta \vec{p}|, \Delta \theta\right.$ and $\left.\Delta|\vec{p}|\right)$ configurations. 

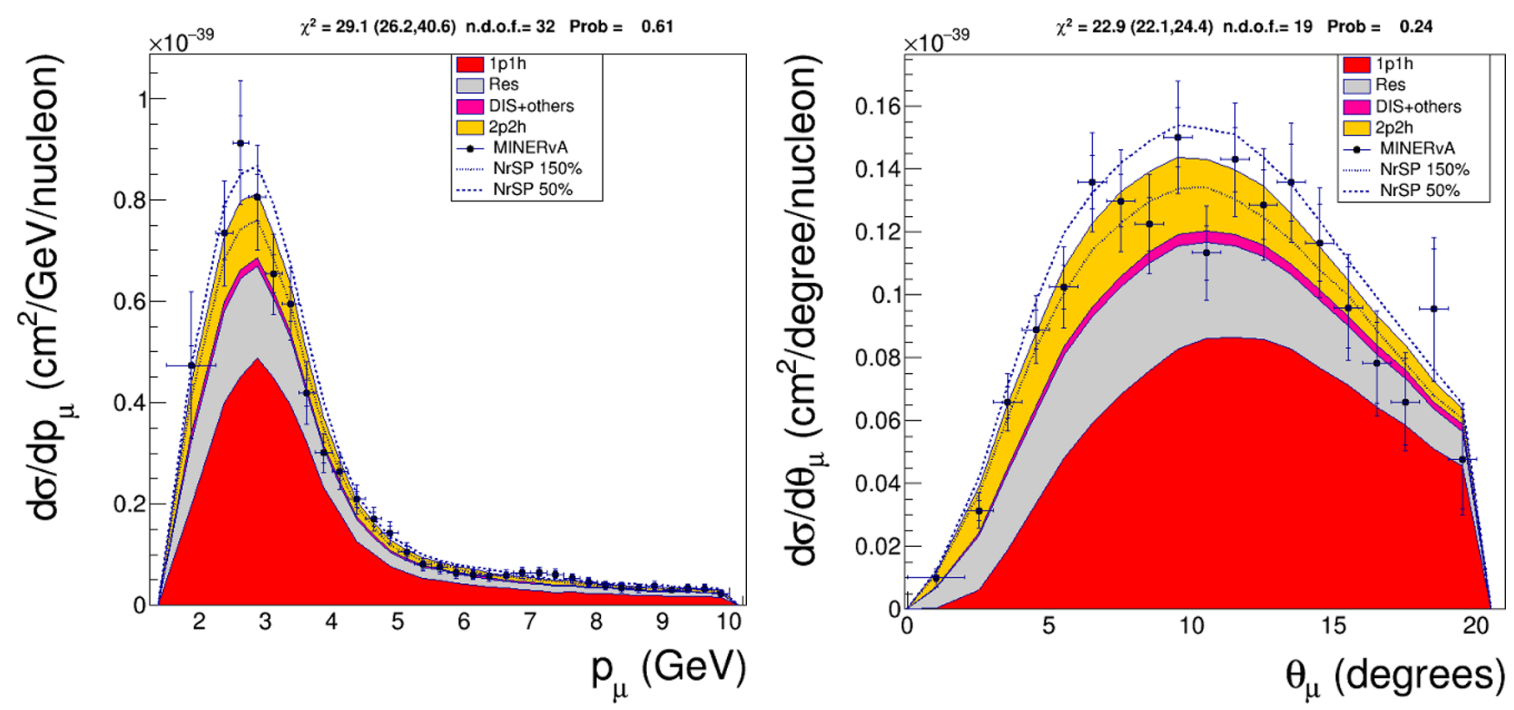

Figure 12. Inclusive $\left|\vec{p}_{\mu}\right|$ (left) and $\theta_{\mu}$ (right) distributions from the CC $0 \pi 1 p$ MINERvA sample [15, 19, 20]. Details of the comparison with NEUT results as in figure 8.
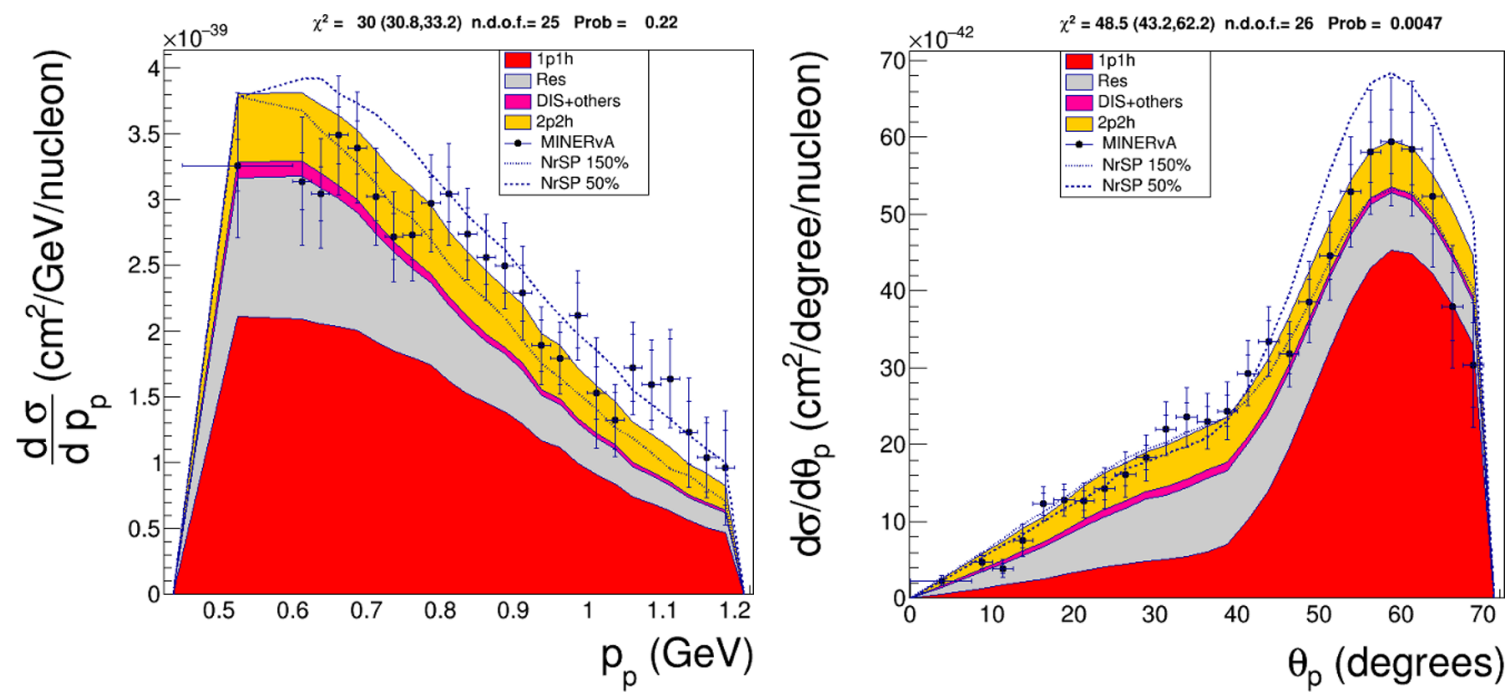

Figure 13. MINERVA CC0 $\pi 1 p$ differential cross sections $[15,19,20]$ in proton kinematics, momentum $\left(\left|\vec{p}_{p}\right|\right)$ and angle $\left(\theta_{p}\right)$, together with results from the current implementation of NEUT. Details of the comparison with NEUT as in figure 8.

The difference between the agreement found for MINERvA and T2K data samples might point to an energy dependent deviation. The LFG model could provide a better approximation to the MINERvA energies than to the T2K ones, which would be more sensitive to finer details of the low-lying nuclear levels. However, an overall $\chi^{2}$-analysis is not sufficient to extract robust conclusions on the energy dependence of the LFG model, and it should also be noted that, as discussed above, MINERvA and T2K data-sets favor different proton re-scattering configurations. This could be an indication that the different agreement exhibited for the MINERvA and T2K data-samples might be also partially pro- 


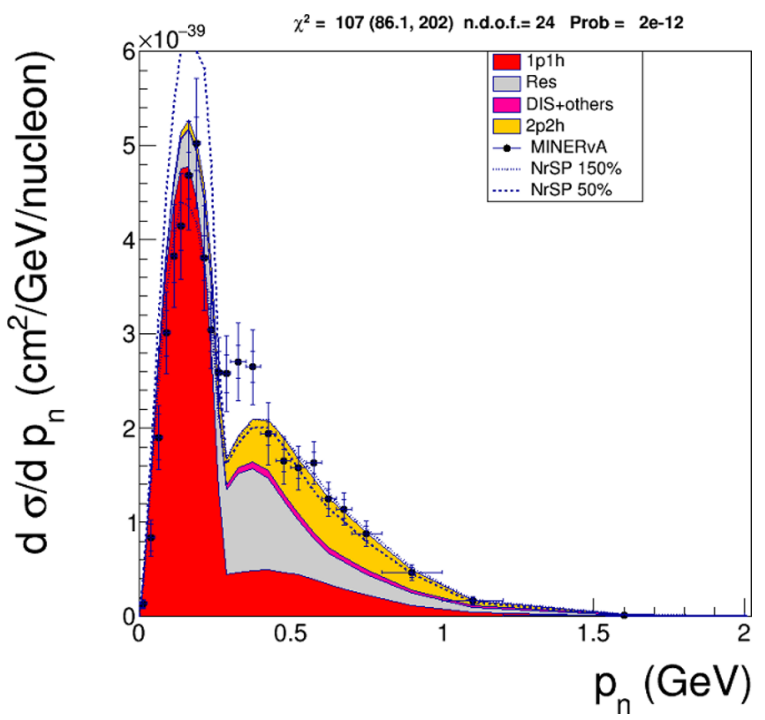

Figure 14. MINERvA CC0 $\pi 1$ p differential cross sections in reconstructed $\left|\vec{p}_{n}\right|$. Data from refs. [15, $19,20]$. We also show results from the current implementation of NEUT, with details of the comparison as in figure 8 .
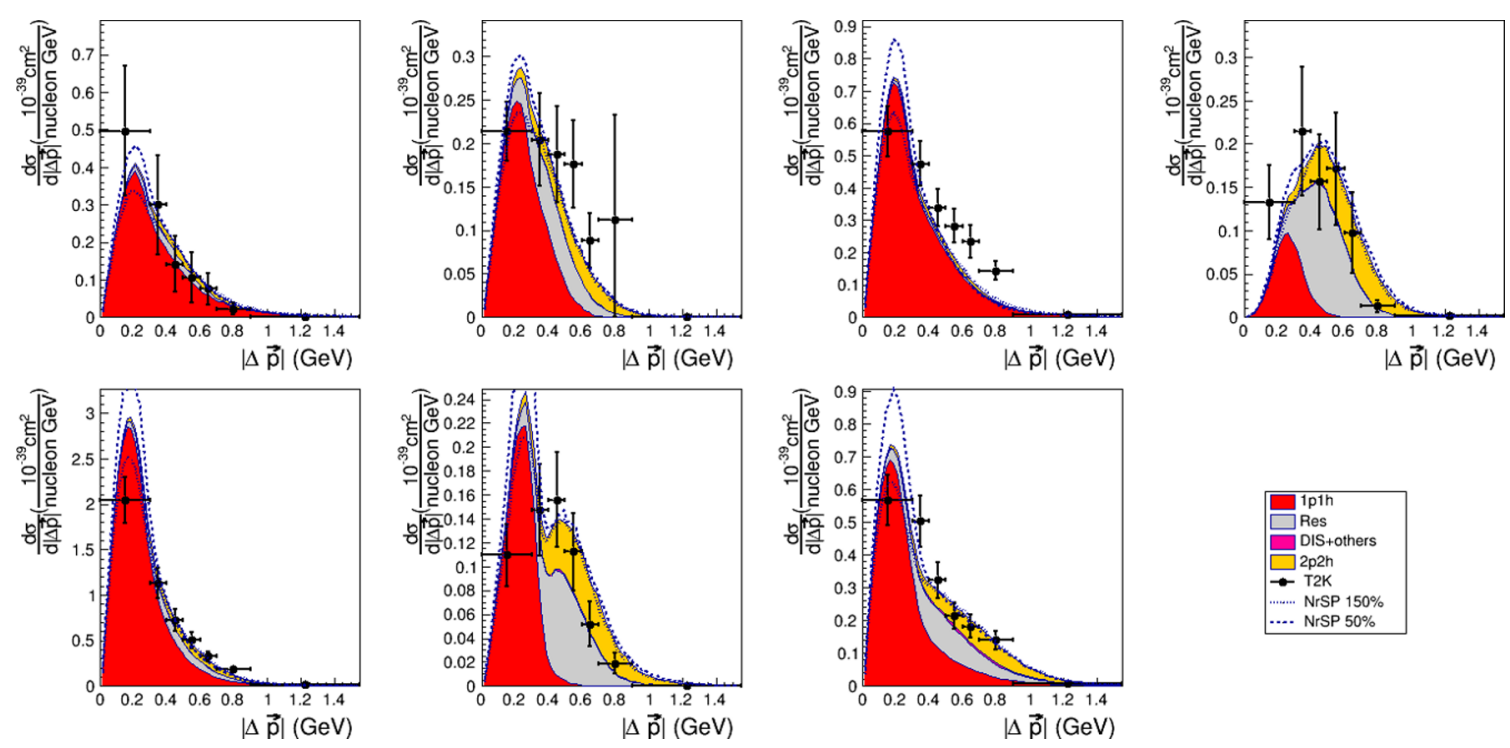

$|\Delta \vec{p}|(\mathrm{GeV})$

Figure 15. T2K CC $0 \pi 1 \mathrm{p}|\Delta \vec{p}|$ distribution. The panels correspond to different muon kinematic bins. From left to right and up to down: $-1<\cos \theta_{\mu}<-0.6,-0.6<\cos \theta_{\mu}<0$ with $\left|\vec{p}_{\mu}\right|<$ $250 \mathrm{MeV},-0.6<\cos \theta_{\mu}<0$ with $\left|\vec{p}_{\mu}\right|>250 \mathrm{MeV}, 0<\cos \theta_{\mu}<1$ with $\left|\vec{p}_{\mu}\right|<250 \mathrm{MeV}, 0<\cos \theta_{\mu}<$ 0.8 with $\left|\vec{p}_{\mu}\right|>250 \mathrm{MeV}, 0.8<\cos \theta_{\mu}<1$ with $250 \mathrm{MeV}<\left|\vec{p}_{\mu}\right|<750 \mathrm{MeV}$ and $0.8<\cos \theta_{\mu}<1$ with $\left|\vec{p}_{\mu}\right|>750 \mathrm{MeV}$. Data taken from ref. [21]. 

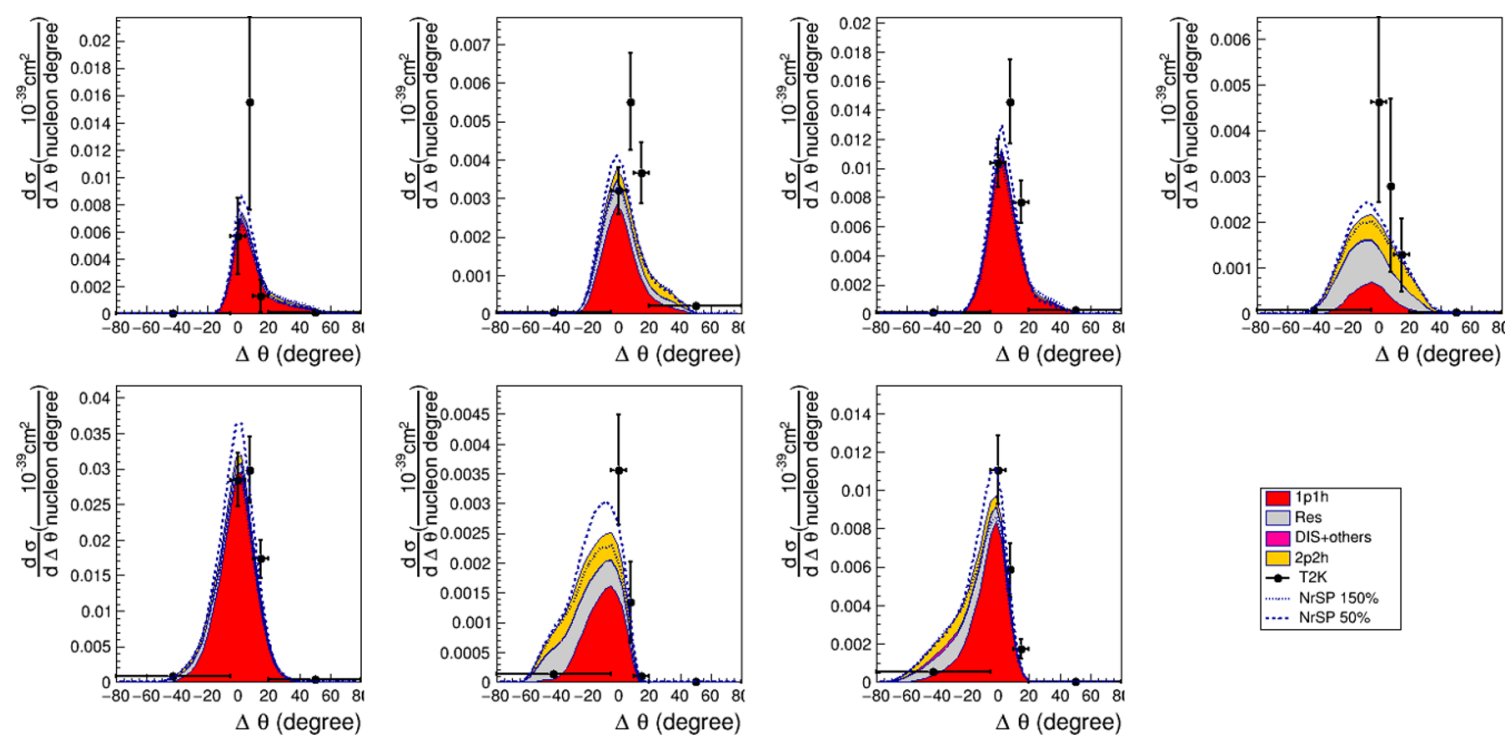

Figure 16. T2K CC $0 \pi 1 \mathrm{p}|\Delta \theta|$ distribution. The panels correspond to the muon kinematic bins specified in figure 15. Data taken from [21].
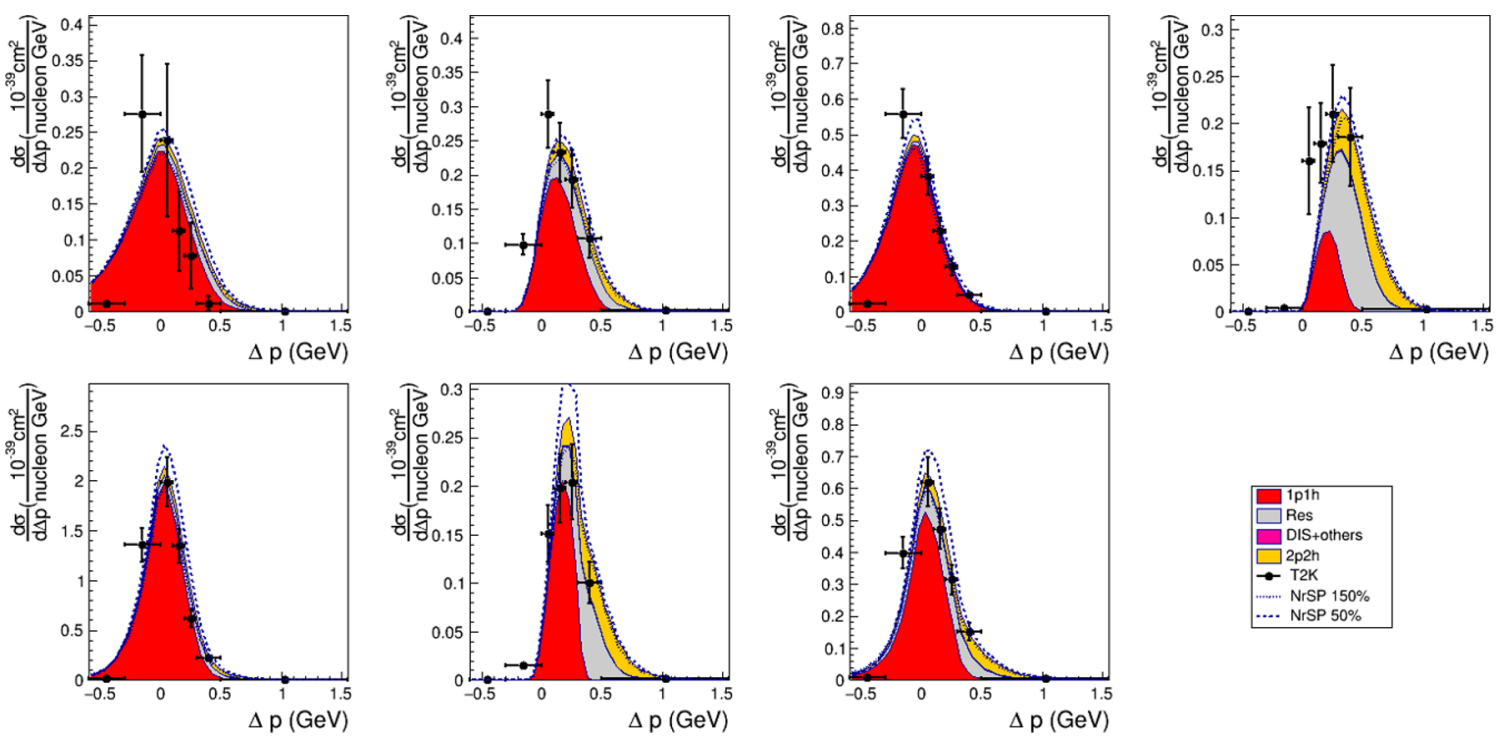

Figure 17. T2K CC0 $01 \mathrm{p} \Delta|\vec{p}|$ distribution. The panels correspond to the muon kinematic bins specified in figure 15. Data taken from [21].

duced by higher energy channels such as $2 \mathrm{p} 2 \mathrm{~h}$ or resonance mechanisms. At this respect, it would be very useful to have access to the T2K $d \sigma / d p_{n}$ cross-section, since this distribution is specially sensitive to non $\mathrm{CC} 1 \mathrm{p} 1 \mathrm{~h}$ contributions, as seen in figure 14 for MINERvA data.

\subsection{Integrated cross-section}

We have performed the numerical integrals of the differential distributions for the six event samples examined in this work. The obtained cross sections, both from data and from the model predictions are compiled in table 5. The results show a common tendency of 


\begin{tabular}{|l|c|c|c|}
\hline & Exp $\left[10^{-39} \mathrm{~cm}^{2}\right]$ & Model $\left[10^{-39} \mathrm{~cm}^{2}\right]$ & $\frac{\text { Exp-Model }}{\text { Model }}[\%]$ \\
\hline \multicolumn{4}{|c|}{ MINERvA } \\
\hline CC inclusive & $18.3 \pm 1.3$ & 16.7 & $9.8 \pm 7.8$ \\
CC0 $\pi$ & $4.64 \pm 0.38$ & 3.96 & $17.1 \pm 9.7$ \\
CC0 $\pi 1 p$ from $\delta \alpha_{T}$ & $1.85 \pm 0.17$ & 1.94 & $-4.6 \pm 9.0$ \\
\hline \multicolumn{4}{|c|}{ T2K } \\
\hline CC inclusive & $7.33 \pm 0.81$ & 6.01 & $22 \pm 13$ \\
CC0 $\pi$ & $4.74 \pm 0.59$ & 3.78 & $25 \pm 16$ \\
CC0 $\pi 1 p$ from $\delta \alpha_{T}$ & $1.39 \pm 0.13$ & 1.46 & $-4.6 \pm 9.0$ \\
CC0 $\pi 1 p$ from $|\Delta \vec{p}|$ & $3.01 \pm 0.34$ & 2.88 & $5 \pm 12$ \\
\hline
\end{tabular}

Table 5. Integrated cross-sections for the different event selections provided by T2K and MINERvA. The CC0 $\pi 1 \mathrm{p}$ integrated cross-section has been computed using the $d \sigma / d\left(\delta \alpha_{T}\right)$ differential distribution for MINERvA and T2K and also $d \sigma / d|\Delta \vec{p}|$ for T2K. In all cases, the nominal NrSP configuration is used.

the NEUT model to predict lower cross-sections for the inclusive and the $\mathrm{CC} 0 \pi$ samples, while its predictions are marginally larger for the $\mathrm{CC} 0 \pi 1 \mathrm{p}$ data-set. The deviations with the theoretical approach for both experiments are similar, except for the case of the CC inclusive. This is expected due to the DIS cross-section, and the very different proportion predicted for this channel for the MINERvA and T2K experiments, see table 6 . The change in the Exp to Model ratio observed from the $\mathrm{CC} 0 \pi$ and the $\mathrm{CC} 0 \pi 1 \mathrm{p}$ may be a consequence of both the proton momentum prediction below the detector detection thresholds and the proton NrSP. The effect of the NRsP on the integrated $\mathrm{CC} 0 \pi 1 \mathrm{p}$ cross-section is shown in table 7 , and it is also included in the proportions collected in table 6 . The number of events with visible protons increases when reducing the NrSP and vice-versa. The effects on MINERvA are slightly reduced with respect to those found for T2K due to the larger proton momentum expected at higher neutrino energies. Even if a reduced NrSP choice will bring the numerical values closer to the differences seen for the $\mathrm{CC} 0 \pi$ samples, we should be cautious. The effect of the CCRes model is not apparent in the results, but however, $23 \%$ in MINERvA and $12.5 \%$ in T2K of the CC0 $\pi 1 \mathrm{p}$ events come from CCRes according to our model. The modelling of the CCRes should also take into account the absorption of the emerging pions by the nucleus.

If, on the contrary, we assume that the CCRes is well simulated, the results point to a deficit in the model prediction of low momentum protons $(\leq 450 \mathrm{MeV})$. A larger re-scattering probability would approach the two results. Another possible cause of the discrepancy is the larger $|\vec{q}|$ values intrinsic to $\mathrm{CC} 0 \pi 1 \mathrm{p}$ with respect to $\mathrm{CC} 0 \pi$ since the proton should be emitted with momentum greater that $450 \mathrm{MeV}$.

Nevertheless, given the experimental uncertainties also included in table 5 , we conclude that the present model implemented in NEUT leads to reasonable integrated cross sections for all six samples considered in this work. 


\begin{tabular}{|l|c|c|c|c|}
\hline & 1p1h (\%) & 2p2h (\%) & Res (\%) & DIS-others (\%) \\
\hline \multicolumn{5}{|c|}{ MINERvA } \\
\hline CC inclusive & 19.0 & 4.7 & 35.4 & 40.9 \\
CC0 $\pi$ & 64.8 & 15.2 & 17.8 & 2.1 \\
CC0 $\pi$ 1p from $\delta \alpha_{T}$ & $56.8_{+1.3}^{-1.4}$ & $16.9_{-0.3}^{+0.2}$ & $23.5_{-1.0}^{+1.1}$ & $2.7_{-0.1}^{+0.2}$ \\
\hline \multicolumn{5}{|c|}{ T2K } \\
\hline CC inclusive & 46.9 & 6.2 & 31.8 & 15.0 \\
CC0 $\pi$ & 78.8 & 10.4 & 10.0 & 0.8 \\
CC0 $\pi 1 p$ from $\delta \alpha_{T}$ & $80.2_{+0.8}^{-1.0}$ & $8.4_{-0.1}^{+0.2}$ & $10.8_{-0.6}^{+0.7}$ & $0.6_{-0.0}^{+0.0}$ \\
CC0 $\pi 1 p$ from $|\Delta \vec{p}|$ & $77.0_{+0.9}^{-1.2}$ & $9.8_{-0.1}^{+0.2}$ & $12.7_{-0.8}^{+0.9}$ & $0.7_{-0.0}^{+0.1}$ \\
\hline
\end{tabular}

Table 6. Different NEUT contributions to the T2K and MINERvA cross sections given in table 5 . As in this latter table, the two T2K CC0 $1 \mathrm{p}$ selections have been considered and all results are obtained using the nominal NrSP. For those samples with explicit proton in the final state $(\mathrm{CC} 0 \pi 1 \mathrm{p})$, we compute the variation of the proportions induced by the use of stronger or weaker proton re-scattering. The differences with respect to the results obtained from the nominal NrSP configuration are shown as an upper (NRsP 150\%) and a lower (NRsP 50\%) error. As expected, the increase (decrease) in the NRsP strength reduces (increases) the population of $\mathrm{CC} 1 \mathrm{p} 1 \mathrm{~h}$ in the sample, affecting indirectly the proportions of the other modes.

\begin{tabular}{|c|c|c|}
\hline $\begin{array}{c}\text { Nominal Model } \\
{\left[\mathrm{cm}^{2}\right]}\end{array}$ & $\begin{array}{c}\text { NRsP 50\% } \\
{\left[\mathrm{cm}^{2}\right]}\end{array}$ & $\begin{array}{c}\text { NRsP 150\% } \\
{\left[\mathrm{cm}^{2}\right]}\end{array}$ \\
\hline \multicolumn{3}{|c|}{ MINERvA } \\
\hline $1.94 \times 10^{-39}$ & $\begin{array}{c}2.09 \times 10^{-39} \\
+7.7 \%\end{array}$ & $\begin{array}{c}1.81 \times 10^{-39} \\
-6.7 \%\end{array}$ \\
\hline \multicolumn{3}{|c|}{$\mathrm{T} 2 \mathrm{~K}$} \\
\hline $1.46 \times 10^{-39}$ & $\begin{array}{c}1.60 \times 10^{-39} \\
+9.6 \%\end{array}$ & $\begin{array}{c}1.35 \times 10^{-39} \\
-7.5 \%\end{array}$ \\
\hline
\end{tabular}

Table 7. T2K and MINERvA CC0 $\pi 1 \mathrm{p}$ total cross-section obtained using NRsP (proton rescattering probability) configurations of $50 \%$ and $150 \%$. We use the $d \sigma / d\left(\delta \alpha_{T}\right)$ differential distribution to perform the numerical integrations. In percentage, we also show the relative variation with respect to the nominal Model.

\section{Data vs theoretical predictions in terms of the scaling variable}

The scaling variable $\psi^{\prime}$ has been shown to be a powerful tool to learn details on the neutrinonucleus interaction [36]. This variable, proposed originally for electron scattering [37, 38], has been adapted to neutrino-nucleus interactions recently. The scaling variable $\left(\psi^{\prime}\right)$ is defined as:

$$
\psi^{\prime}=\frac{1}{\sqrt{\sqrt{1+\eta_{F}^{2}}-1}} \frac{\lambda-\tau}{\sqrt{(1+\lambda) \tau+\kappa \sqrt{\tau(1+\tau)}}}
$$


with

$$
\eta_{F}=\frac{p_{F}}{m_{n}}, \kappa=\frac{|\vec{q}|}{2 m_{n}}, \lambda=\frac{q^{0}-E_{\text {shift }}}{2 m_{n}} \text { and, } \tau=\kappa^{2}-\lambda^{2}
$$

where, in addition to variables already introduced, $p_{F}$ is the Fermi momentum and $E_{\text {shift }}$ is a energy shift, which are fixed in this discussion to $228 \mathrm{MeV}$ and $20 \mathrm{MeV}$, respectively.

The scaling variable $\psi^{\prime}$ allows to project the energy-momentum transfer $\left(q^{0},|\vec{q}|\right) 2 \mathrm{D}$ sample of events into a 1D distribution, as illustrated in figure 18 with the NEUT predictions for the CC inclusive MINERvA sample. The full 2D distribution is shown in the left plot, where we also display the $\psi^{\prime}$-constant curves. We see that a $\left(q^{0},|\vec{q}|\right)$ pair determines an unique $\psi^{\prime}$ value, while the same scaling variable can be constructed out of different energy-momentum transfer combinations. The $\psi^{\prime}$-distribution of events is depicted in the right panel. Though $\psi^{\prime}$ is based on muon kinematics, the underlying contributions from different mechanisms (1p1h, 2p2h Res and DIS) are better separated since they lead to different $\psi^{\prime}$-behaviors, as can be appreciated in the figure. Hence, this variable might provide a method to effectively disentangle the components of nuclear effects in data and therefore extract valuable constraints on the theoretical model ingredients. This is the objective of the new analysis proposed in this work, and the details of which are discussed below.

The scaling variable $\psi^{\prime}$ is not accessible to traditional neutrino experiments, since the neutrino energy is not measurable in an event by event basis to compute momentum and energy transfers. Neutrino experiments usually report their flux-averaged cross-section results as function of muon-kinematics bins, ${ }^{8} d \sigma_{\operatorname{Exp}} / d \vec{p}_{\mu}$, which can be compared with theoretical MC differential distributions for the same binning,

$$
R\left(\vec{p}_{\mu}\right)=\frac{d \sigma_{\mathrm{Exp}} / d \vec{p}_{\mu}}{d \sigma_{\mathrm{MC}} / d \vec{p}_{\mu}}
$$

On the other hand within the theoretical MC model, one can associate each event with a value of the scaling variable $\psi^{\prime}$ (in general, values of the scaling variable comprised in a certain bin, since both neutrino energy and muon-kinematics are binned). We propose to express the ratio of data to theoretical MC predictions (data/MC, in what follows) as function of $\psi^{\prime}$. Thus, we define the average data/MC ratio as:

$$
\begin{aligned}
R\left(\psi^{\prime}\right) & =\sum_{\text {events MC } \in \psi^{\prime}} \sum_{p_{\mu} \theta_{\mu}} f\left(\vec{p}_{\mu} \mid \psi^{\prime}\right) R\left(\vec{p}_{\mu}\right) \\
f\left(\vec{p}_{\mu} \mid \psi^{\prime}\right) & =\frac{N_{\mathrm{MC}}\left(\vec{p}_{\mu} \mid \psi^{\prime}\right)}{N\left(\psi^{\prime}\right)}
\end{aligned}
$$

with $N\left(\psi^{\prime}\right)$ the number total of MC generated events, and $N_{\mathrm{MC}}\left(\vec{p}_{\mu} \mid \psi^{\prime}\right)$ the number of events with muon kinematics comprised in the bin around $\vec{p}_{\mu}$, which gives rise to the value $\psi^{\prime}$ for the scaling variable. ${ }^{9}$ Thus, $f\left(\vec{p}_{\mu} \mid \psi^{\prime}\right)$ is the fraction of events predicted by the theoretical model for a given value of $\psi^{\prime}$ and muon kinematics $\vec{p}_{\mu}$, taking into account the neutrino energy spectrum of the experiment. The distribution $R\left(\psi^{\prime}\right)$ is built in such a way that the

\footnotetext{
${ }^{8}$ Experiments normally report $\left(\left|\vec{p}_{\mu}\right|, \theta_{\mu}\right)$ or $\left(p_{\|}, p_{\perp}\right)$.

${ }^{9}$ Note that eq. (5.4) admits a matrix interpretation of the type $B=M A$, where $A$ and $B$ are ratio vectors and $M$ is a matrix relating $\psi^{\prime}$ and the kinematics of the observed muon.
} 

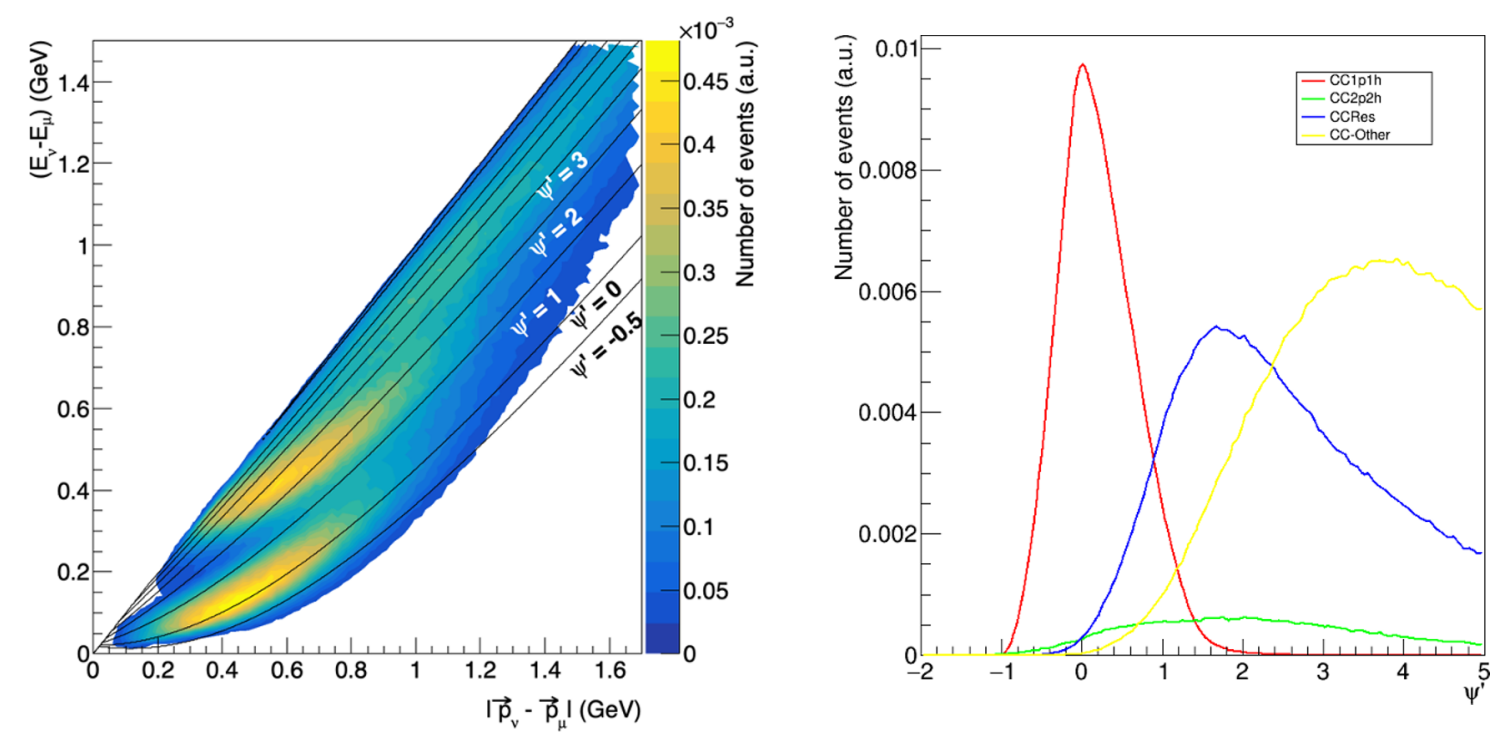

Figure 18. Left: CC inclusive MINERvA $\left[|\vec{q}|=\left|\vec{p}_{\nu}-\vec{p}_{\mu}\right|, q^{0}=\left(E_{\nu}-E_{\mu}\right)\right]$ 2D distribution predicted by the NEUT CC inclusive event generator. The black solid lines mark fix $\psi^{\prime}$ values across the $\left(q^{0},|\vec{q}|\right)$-plane. Right: $\psi^{\prime}$ distribution of events obtained from the $2 \mathrm{D}$ one shown in the left-panel, and separated by the primary neutrino-nucleon interaction modes.

ratio between data and the theoretical MC results are weighted according to the population $f\left(\vec{p}_{\mu} \mid \psi^{\prime}\right)$. In the limit in which $f=1$ for one kinematics-bin and there is no more than one value of $\psi^{\prime}$ contributing to this bin, $R$ is no more than the ratio of data to MC for this given value of $\psi^{\prime}$. This is not the case in most of the experimental bins, but we still expect that some of the deviations from measurements are accumulated in the corresponding value of the scaling variable.

In addition, the number of events $N_{\mathrm{MC}}\left(\vec{p}_{\mu} \mid \psi^{\prime}\right)$ can be split into the different mechanisms $(1 \mathrm{p} 1 \mathrm{~h}, 2 \mathrm{p} 2 \mathrm{~h}$, Res, DIS, ... ) considered in the theoretical approach implemented in the MC. In figure 19, we illustrate the physics content of the 3D $f\left(\vec{p}_{\mu} \mid \psi^{\prime}\right)$ transfer matrix for the CC inclusive MINERvA sample, as predicted by NEUT. In the figure, we show the number of events $N_{\mathrm{MC}}\left(\vec{p}_{\mu} \mid \psi^{\prime}\right)$, accumulated for different $\psi^{\prime}$ intervals. As expected from the top panel of figure 6 , the largest contributions to $N_{\mathrm{MC}}\left(\vec{p}_{\mu} \mid \psi^{\prime}\right)$, for all $\psi^{\prime}$-regions, are concentrated in the two dimensional region $\left[1.5 \mathrm{GeV}<p_{\|}<4 \mathrm{GeV}\right] \times\left[0.25 \mathrm{GeV}<p_{\perp}<1 \mathrm{GeV}\right]$. However, the relative contributions of the different interaction modes $(\mathrm{CC} 1 \mathrm{p} 1 \mathrm{~h}, \mathrm{C} 2 \mathrm{p} 2 \mathrm{~h}, \mathrm{CCR}$ es and CCOthers) change significantly with the $\psi^{\prime}$-bin, following a pattern consistent with the distribution displayed in the right plot of figure 18.

We stress the trivial observation that a $\left(p_{\|}, p_{\perp}\right)$ pair does not unequivocally determine a value of $\psi^{\prime}$, as clearly illustrated in figure 19. This is because the neutrino beam is not monochromatic, and for each neutrino energy one has a different relation between the $\left(p_{\|}, p_{\perp}\right)$ and $\left(q^{0},|\vec{q}|\right)$ pairs. ${ }^{10}$ As a consequence in a $\left(p_{\|}, p_{\perp}\right)$ distribution, a fix value of $\psi^{\prime}$ will not be represented by a curve, but instead by a $2 \mathrm{D}$ region, with large overlaps

\footnotetext{
${ }^{10}$ One has $q^{0}=E_{\nu}-\sqrt{m_{\mu}^{2}+p_{\|}^{2}+p_{\perp}^{2}}$ and $\vec{q}^{2}=\left(E_{\nu}-p_{\|}\right)^{2}+p_{\perp}^{2}$.
} 

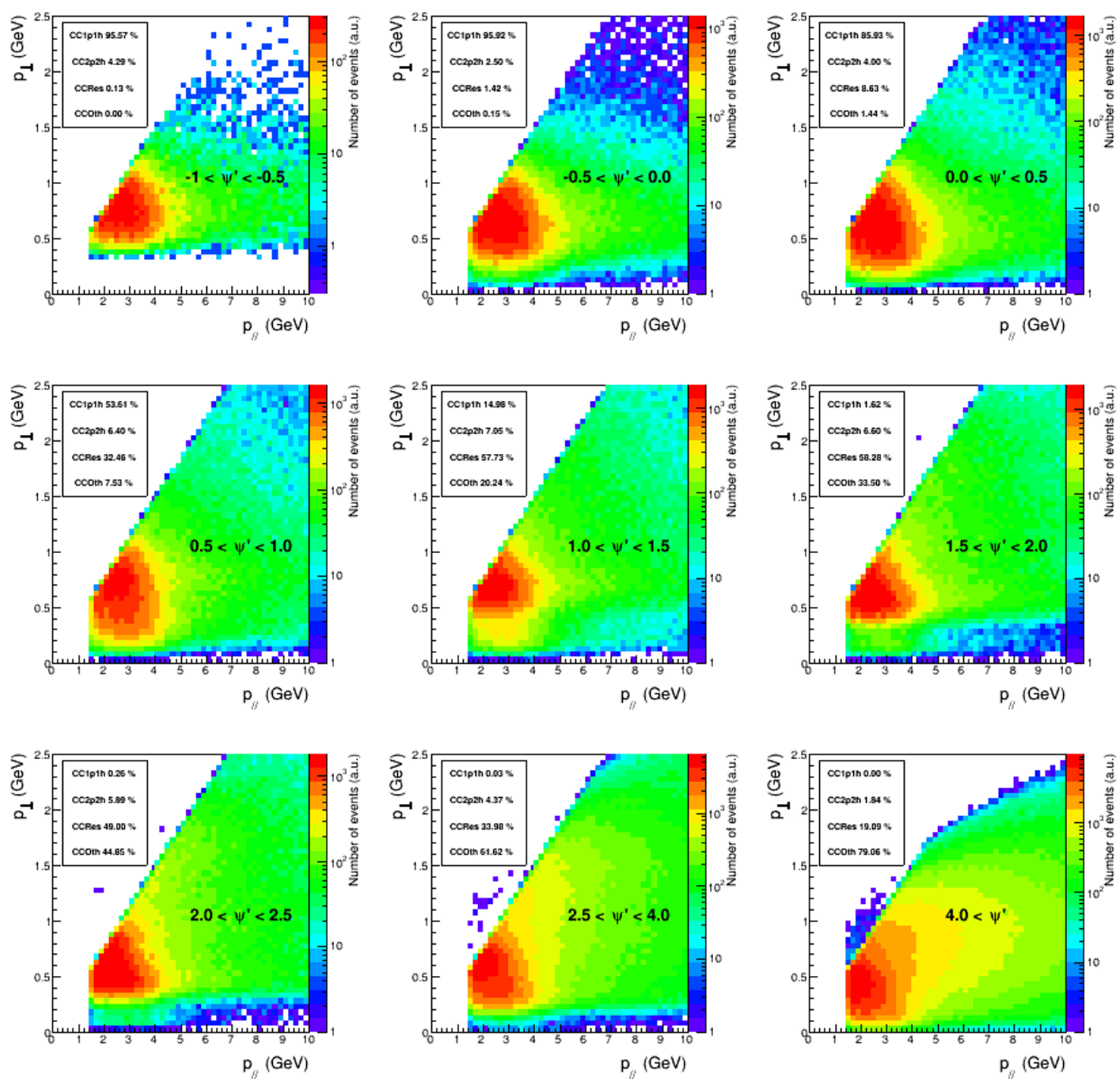

Figure 19. Two dimensional $\left(p_{\|}, p_{\perp}\right)$ lepton distributions accumulated for different $\psi^{\prime}$-intervals from the NEUT CC inclusive event generator for MINERvA flux. The contributions (in percentage) of the different interaction modes (CC1p1h, C2p2h, CCRes and CCOthers) are also shown in each of the panels.

between different $\psi^{\prime}$-regions. We should note, related to this discussion, that the total number of events for a given $\psi^{\prime}\left[N\left(\psi^{\prime}\right)\right.$ in the definition of $f\left(\vec{p}_{\mu} \mid \psi^{\prime}\right)$ in eq. (5.5)] receives sizable contributions from a whole interval of neutrino energies of the incoming broad beam. This is shown in the upper plots of figure 20 for both MINERvA and T2K CC inclusive samples. We see that for this selection, the latter experiment is much more dominated by the $1 \mathrm{p} 1 \mathrm{~h}$ reaction mechanism (region of $\psi^{\prime}$ around one) than MINERvA, for which resonant and DIS modes, located at higher values of the scaling variable, become more relevant. Moreover, the dispersion of neutrino energies for MINERvA is quite significant, while the high-energy tail for T2K is less important. In the lower plots of figure 20, we show the corresponding column (energy-bin) normalized distributions, where the effect of 

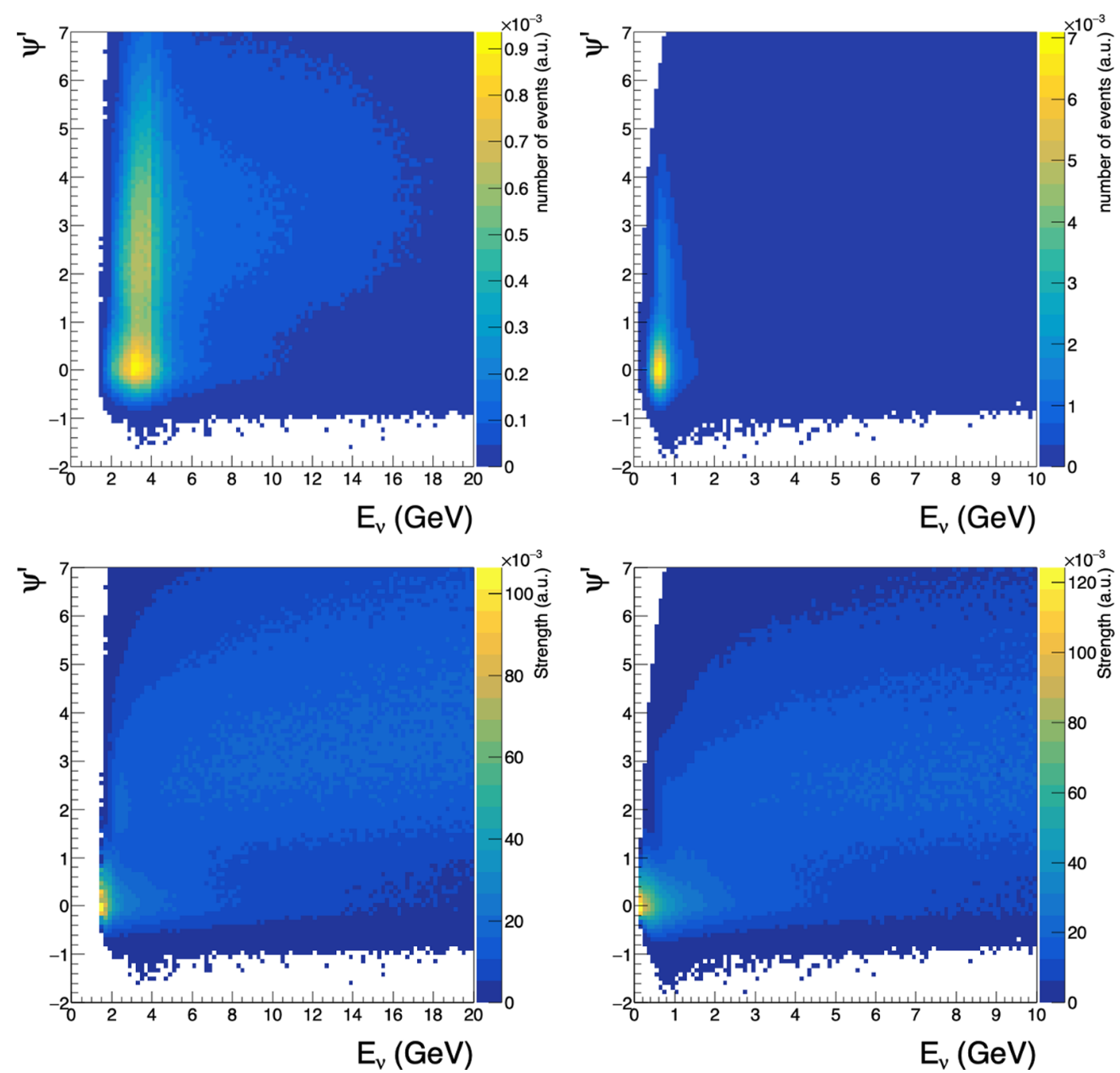

Figure 20. Top: distribution of the scaling variable $\psi^{\prime}$ versus the neutrino energy for the CC inclusive MINERvA (left) and T2K (right) experiments, as predicted by NEUT. Bottom: the distributions shown in the upper panels, but normalised to unity by column (energy-bin).

the neutrino flux times the neutrino cross-section should largely cancel. We observe an almost universal pattern, which would be the conditional probability $P\left(\psi^{\prime} \mid E_{\nu}\right)$, corrected only by the detector acceptance effects that are clearly seen at neutrino energies between $0.2 \mathrm{GeV}$ and $3 \mathrm{GeV}$.

The errors on $R\left(\psi^{\prime}\right)$ and the covariance matrix for different values of $\psi^{\prime}$ can be propagated using the above definition and the covariance matrix from the experiments. We have applied this algorithm to T2K and MINERvA inclusive and $\mathrm{CC} 0 \pi$ cross-sections.

\subsection{Results for $R\left(\psi^{\prime}\right)$}

Minerva CC inclusive $R\left(\psi^{\prime}\right)$ is shown in the left panel of figure 21, where the contributions of the different reaction channels are also shown. The total distribution shows a remarkable 

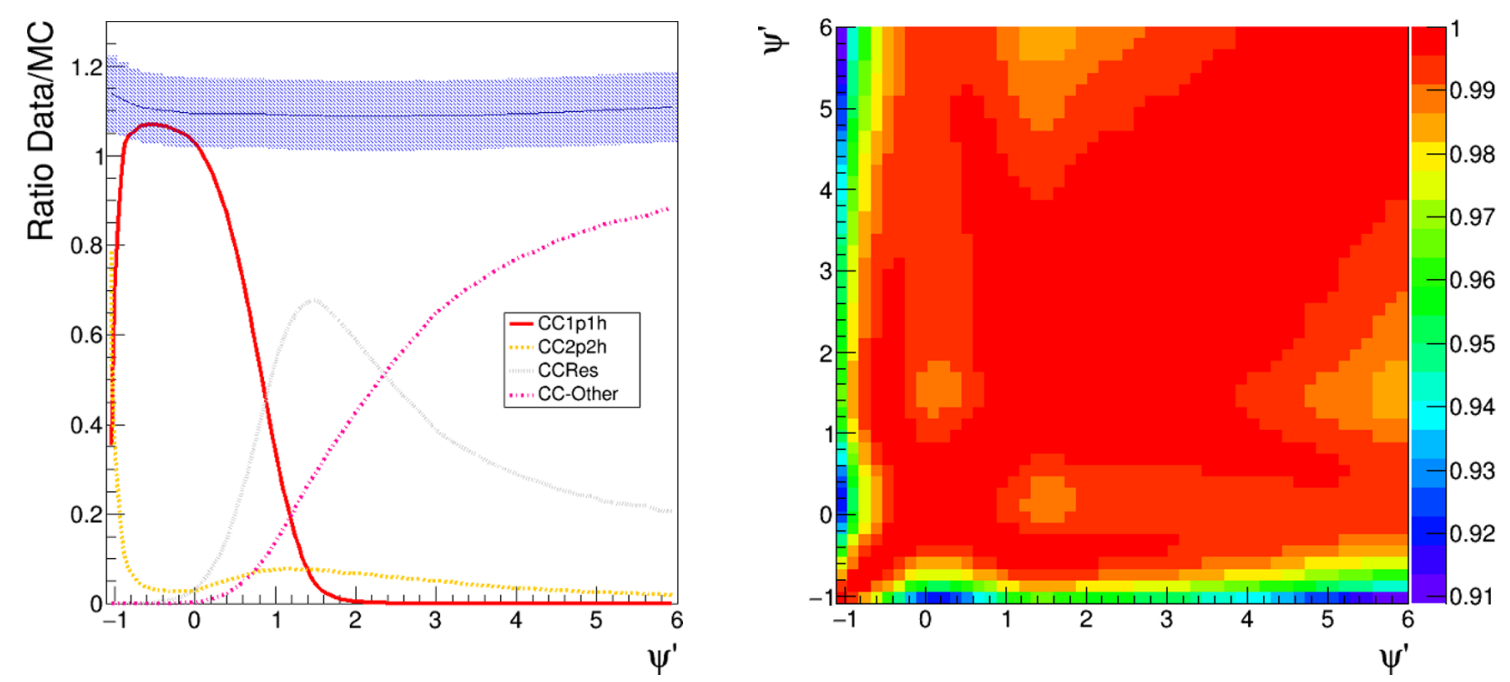

Figure 21. Left: Data/MC ratio $R\left(\psi^{\prime}\right)$ calculated for the MINERvA CC inclusive cross-section, and split into the CC1p1h (red), CC2p2h (yellow), CCRes (gray) and CC-DIS (pink) contributions. Right: $R\left(\psi^{\prime}\right)$ correlation matrix computed for the MINERvA CC inclusive cross-sections.
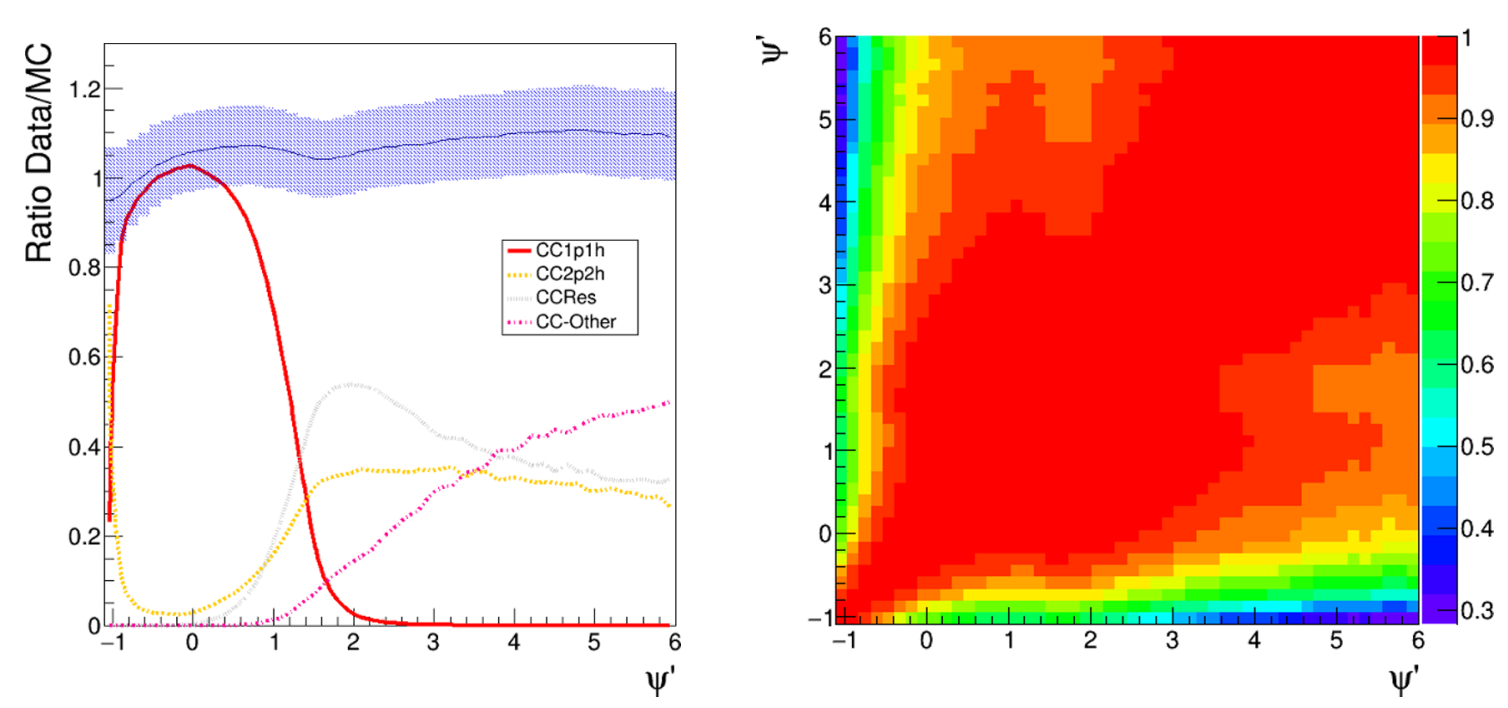

Figure 22. The same as figure 21, but for the MINERvA CC0 $\pi$ cross-section.

pattern, with the ratio almost constant, independent of $\psi^{\prime}$, around 1.1 (i.e. the MC prediction is some $10 \%$ lower than the data). This means that the proportion of the different reaction channels is well balanced. The $2 \mathrm{p} 2 \mathrm{~h}$ contribution is very small and it is difficult that this mechanism could significantly influence the overall picture, but both $1 \pi$ and DIS channels smoothly balance above $\psi^{\prime}=1$. We show the $R$-correlation matrix in the right plot of figure 21. The correlation is larger than $90 \%$ for any pair of $\psi^{\prime}$ values. This is a consequence of both the initial correlation between experimental results and the fact that several $\psi^{\prime}$-values contributes to the same muon-kinematics bin. 

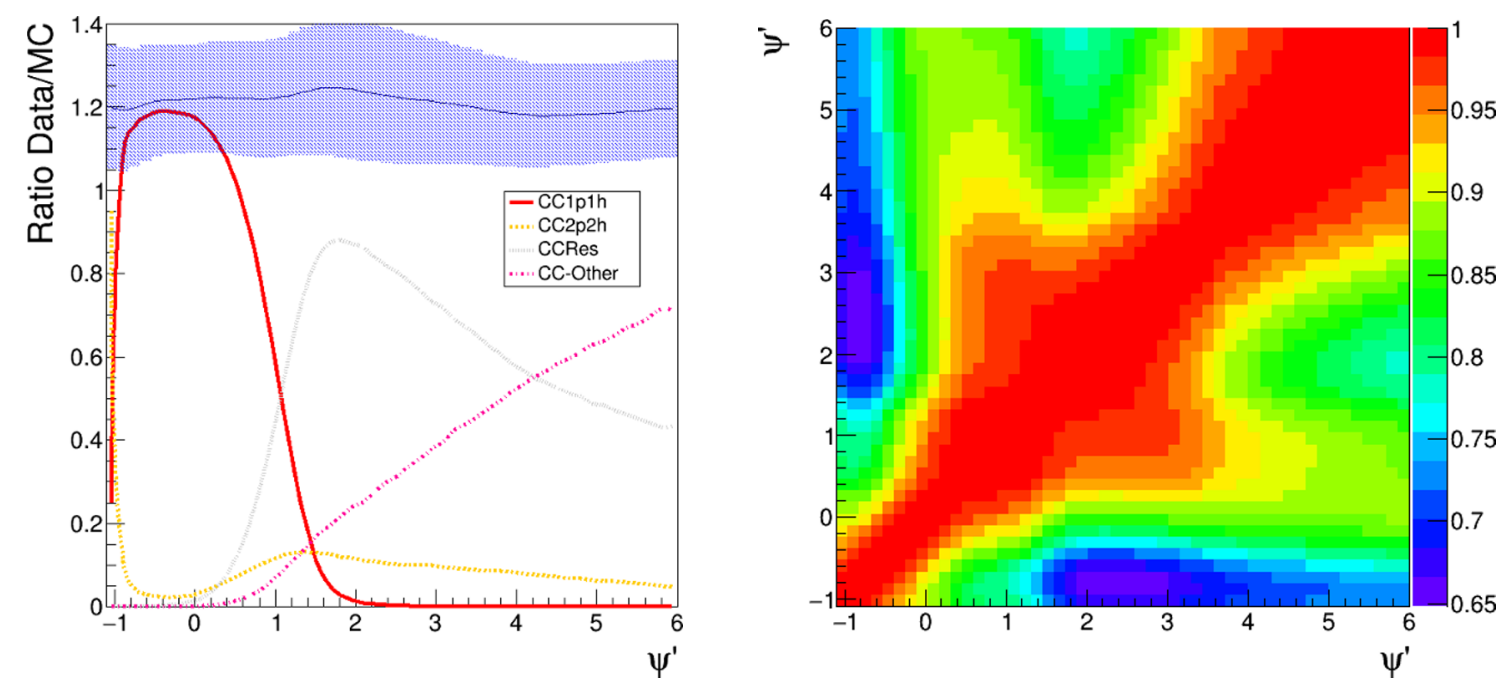

Figure 23. The same as figure 21, but for the T2K CC inclusive cross-section.
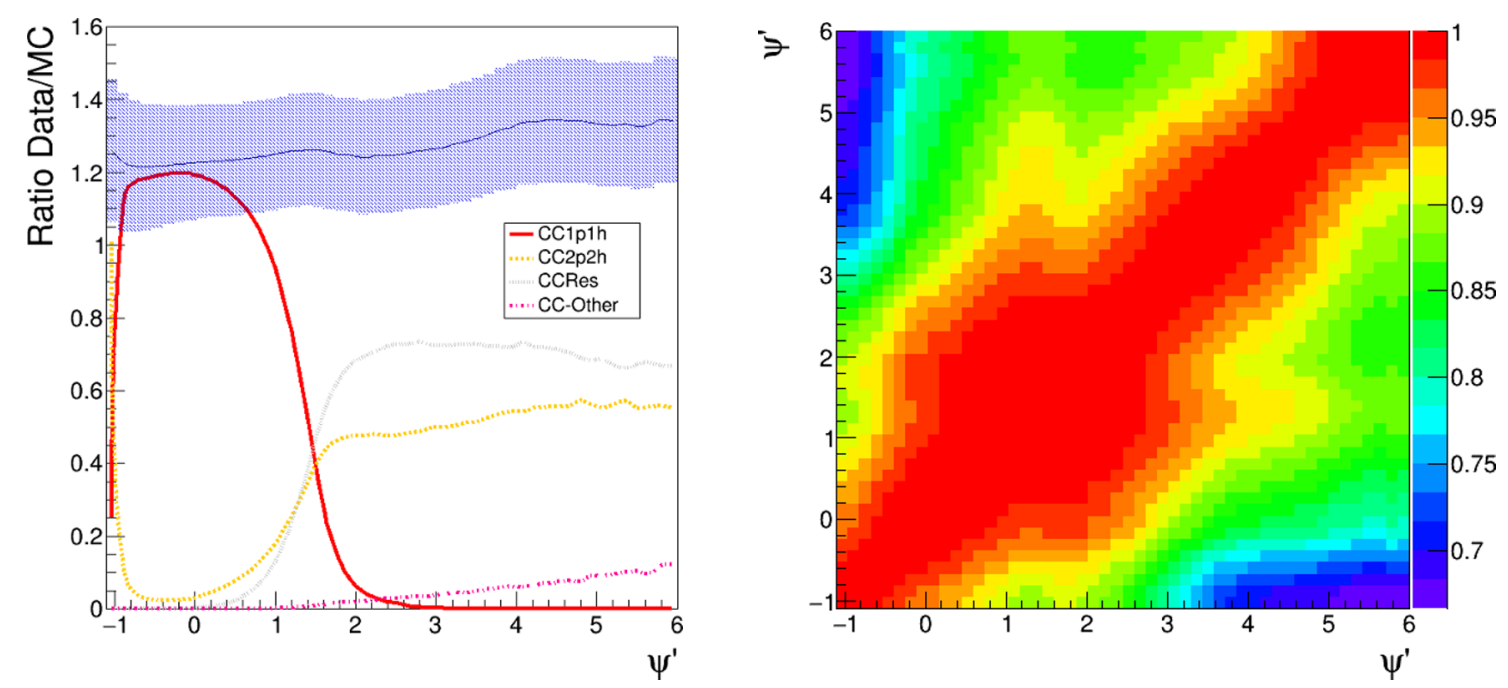

Figure 24. The same as figure 21 , but for the $\mathrm{T} 2 \mathrm{~K} \mathrm{CC} 0 \pi$ cross-section.

We repeat in figure 22 the exercise with the $\mathrm{CC} 0 \pi$ MINERvA data. The tendency observed is similar to that seen for the CC inclusive. MC predicts smaller cross-section by a similar amount (10\%), though in the $\mathrm{CC} 0 \pi$ case some structure is observed with a small deficit of MC below $\psi^{\prime}=0$. On the contrary, the region above $\psi^{\prime}=2$ shows an excess on the MC predictions with respect to data. This region is dominated by $2 \mathrm{p} 2 \mathrm{~h}$ and $1 \pi$ (Res) channels. It is important to notice that these results do not call for a large modification of the $2 \mathrm{p} 2 \mathrm{~h}$ contribution as requested by the calorimetric measurements in MINERvA [29]. The bin to bin correlation is shown in the right panel of the figure. The observed correlations are smaller than in the previous case, with some regions below $70 \%$. This might explain the appearance of some structure in the $\mathrm{CC} 0 \pi$ ratio plot. 
The $R\left(\psi^{\prime}\right)$ results for T2K CC inclusive and $\mathrm{CC} 0 \pi$ samples are shown in figures 23 and 24, respectively. The observed patterns in both cases are similar to those discussed above for the MINERvA experiment. Our model predicts smaller cross-section than the measurements by about $20 \%\left(R\left(\psi^{\prime}\right) \sim 1.2\right)$, while the distribution of the ratio as function of $\psi^{\prime}$ is again rather flat. There is some reflection of the CCRes contribution in figure 23 around $\psi^{\prime} \sim 1.5$, which might be an indication of an even smaller single pion cross-section. Nevertheless and despite the large correlations, the evidence is weak, given the flat behavior of the ratio and the large errors. Similar low cross-section predictions were also observed in the T2K CC1 $\pi$ results [14]. The impact of the possible $\mathrm{CC} 1 \pi$ miss-modelling is minimised in the case of $\mathrm{CC} 0 \pi$ where the contribution of $\mathrm{CC} 1 \pi$ becomes as the same level than $\mathrm{CC}$ $2 \mathrm{p} 2 \mathrm{~h}$, see table 6 . The region around the CCQE peak shows a flat dependency with the scaling variable contrary to the MINERvA $\mathrm{CC} 0 \pi$ results which exhibit a visible decrease below $\psi^{\prime}<0$. It is also observed that the prediction is balanced between the different reaction channels. The correlation matrices are shown in the right panels of figures 23 and 24. The correlations for both $\mathrm{CC}$ inclusive and $\mathrm{CC} 0 \pi$ are smaller than in the case of MINERvA, $60 \%$ correlation between values of $\psi^{\prime}$ below 0 and higher than 1.5. This reduced correlation gives more credibility to the tendencies of $R\left(\psi^{\prime}\right)$.

The large errors $(\approx 10 \%$ for $\mathrm{T} 2 \mathrm{~K}$ and $\approx 5 \%$ for MINERvA) are the consequence of large positive correlations enhancing the experimental errors.

To understand the large correlations across all values of $\psi^{\prime}$ both for T2K and MINERvA, we estimated the correlation removing all the off-diagonal terms in the covariance matrices provided by the experiment. In this case, the ratios $R\left(\psi^{\prime}\right)$ for different values of the scaling variable can be correlated only because receive contributions from the same muon-kinematics bin for different neutrino energies, contained in the non-monochromatic beam (see the discussion above of figures 19 and 20). The smallest observed correlation is reduced from $90 \%$ to $40 \%$ in the case of MINERvA and from $60 \%$ to $5 \%$ in the case of $\mathrm{T} 2 \mathrm{~K}$. The better figure in the $\mathrm{CC} 0 \pi \mathrm{T} 2 \mathrm{~K}$ sample might be a consequence of the narrow neutrino beam at $\mathrm{T} 2 \mathrm{~K}$, which allows to separate better the regions dominated by $1 \mathrm{p} 1 \mathrm{~h}$ and the resonant and DIS components. The experimental correlations errors come mostly from flux uncertainties, statistical and systematic experimental errors but also from bin to bin migrations in the extraction of the cross-section. Improvement in flux determination, larger statistics to reduce the bin size and select the proper data representation might improve the conclusions of this study.

\section{Conclusions}

We have presented an exclusive final state model to describe $\mathrm{CC} 1 \mathrm{p} 1 \mathrm{~h}$ interactions. The approach is based on a LFG picture of the nucleus and uses a consistent implementation of the removal energy, that provides an estimation of the excitation of the final nuclear system. The model has been included in NEUT to profit from the existing simulation of $2 \mathrm{p} 2 \mathrm{~h}$, pion production and DIS mechanisms and on the transport simulation of the hadrons inside the nucleus after the interaction. Predictions are simultaneously compared to the most recent $\mathrm{T} 2 \mathrm{~K}$ and MINERvA inclusive, $\mathrm{CC} 0 \pi$ and TKI variable results, showing 
an acceptable agreement with the data from both experiments. Results from T2K suffer from low statistics, but they also show worse agreement with the model predictions. This might be an indication of some energy dependency that is not properly accounted by this implementation. The correct modelling of the energy removal reduces the amount of interactions at low $q^{2}$ and the total CC1p1h cross-section facilitating the agreement with the experimental results. On the other hand, the overall good description of the MINERvA TKI variables found here and, in general of its $\mathrm{CC} 0 \pi$ data-sample, does not support a large modification of the $2 \mathrm{p} 2 \mathrm{~h}$ contribution as requested by the calorimetric measurements of that collaboration [29]. The latter conclusion agrees with the findings in ref. [15] with NuWro [39] and GiBUU [40] event generators (GiBUU results can be found in the Supplemental Material for ref. [41]). However, we should point out that the re-weight of the $2 \mathrm{p} 2 \mathrm{~h}$ strength proposed in [29] is based on the inclusive sample, which is a superset of the $\mathrm{CC} 0 \pi$ and $\mathrm{CC} 0 \pi 1 \mathrm{p}$ data-sets considered in this work.

We have also proposed a novel comparison between flux-folded data and MC theoretical predictions accumulated in bins of the scaling variable $\Psi^{\prime}$, which can be used to signal possible deficiencies of theoretical schemes.

A microscopic interpretation of the relevant reaction mechanisms becomes essential in neutrino oscillation experiments in order to achieve a correct reconstruction of the incoming neutrino kinematics, free of conceptual biasses. Studies, as the one presented in this work, are of the utmost importance for the ambitious experimental program which is underway to precisely determine neutrino properties, test the three-generation paradigm, establish the order of mass eigenstates and investigate leptonic CP violation.

\section{A Comparison of momentum and energy transfer distributions}

The different experimental selection criteria might bias the momentum and energy transfers. The comparison of the different accessible $\left(q^{0},|\vec{q}|\right)$-phase space provide some indications of possible deficits in the models.

The model estimation for the energy and momentum transfer distributions for the MINERvA and T2K CC inclusive event selections are shown in figure 25. The cut off in $|\vec{q}|$ implemented in the CC2p2h model is clearly visible in the MINERvA distributions. The CC2p2h cross-section at the cutoff $|\vec{q}|=1.3 \mathrm{GeV}$ represents around $10 \%$ of the total one for this momentum transfer. Although, we find similar drops for MINERvA and T2K, there are more contributions to the cross-section in the first experiment above $|\vec{q}|=1.3 \mathrm{GeV}$, and hence we expect that the implementation of this cutoff to make a bigger impact in the total cross-section determination for MINERvA. As expected, the resonant, $2 \mathrm{p} 2 \mathrm{~h}$ and DIS contributions are larger for the MINERvA energies, see table 6 .

The predicted energy and momentum transfer distributions for the MINERvA and $\mathrm{T} 2 \mathrm{~K} \mathrm{CC} 0 \pi$ event-selections are shown next in figure 26 . As in the previous case, the cut off $|\vec{q}| \leq 1.3 \mathrm{GeV}$ for the CC2p2h contribution is clearly visible, both for MINERvA and $\mathrm{T} 2 \mathrm{~K}$ distributions, with the drop at the cutoff amounting around $10 \%$ of the total cross-section As expected, the $\mathrm{CC} 0 \pi$ event-sample has smaller contamination from DIS and resonant processes, which leads to a small bias in the event selection. Moreover, the 

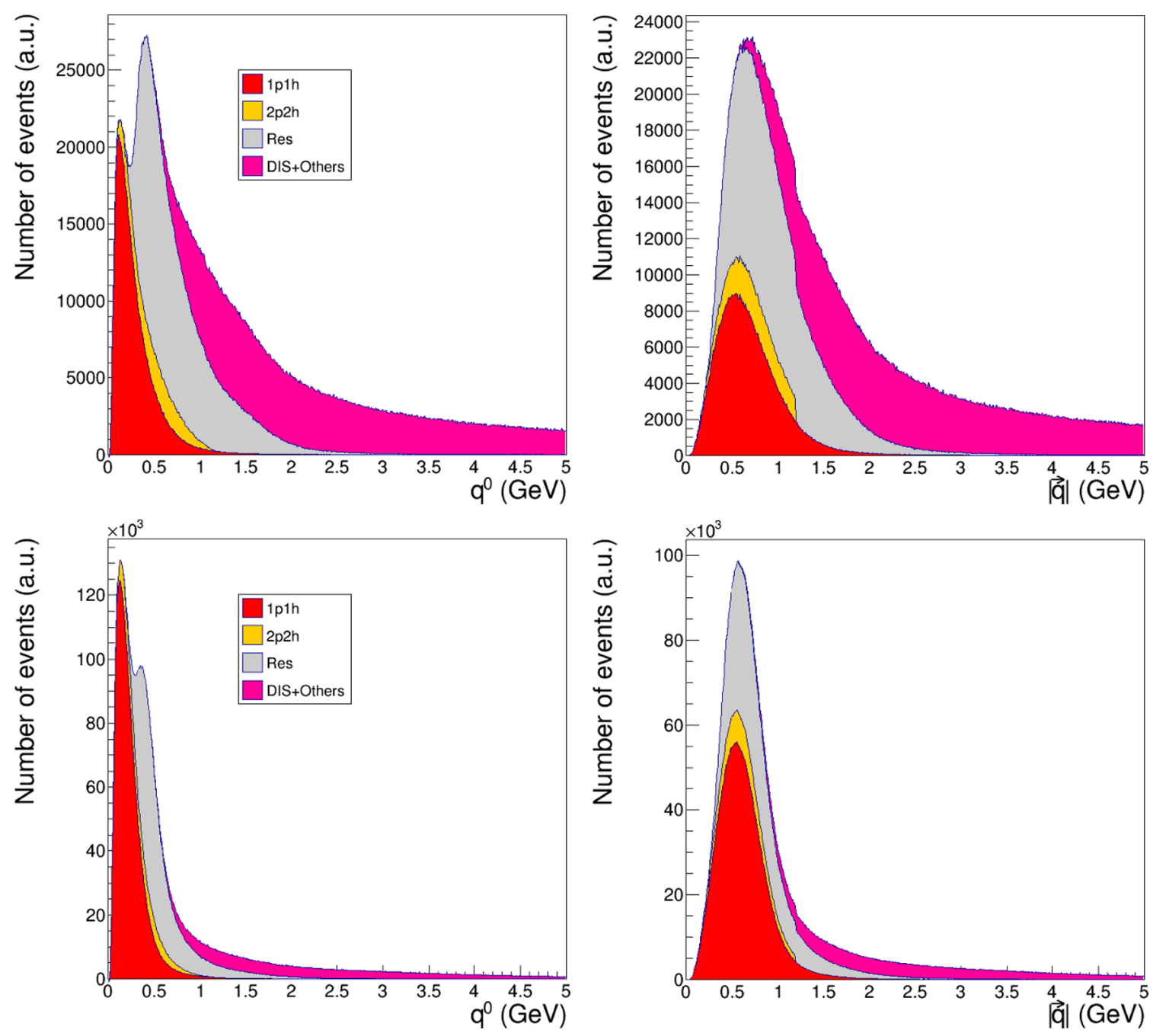

Figure 25. MINERvA (up) and T2K (bottom) energy $q^{0}$ (left) and momentum $|\vec{q}|$ (right) transfer distributions for the CC inclusive selections.

CC1p1h contributions in this data selection show a dependence on $q^{0}$ and $|\vec{q}|$ similar as that observed for the $\mathrm{CC}$ inclusive ones.

The $q^{0}$ and $|\vec{q}|$ event distributions used for the TKI variable analysis (CC0 $\left.\pi 1 \mathrm{p}\right)$ are shown in figure 27. The significant reduction of the CCRes and CCOther contributions is evident in the plots and it can be also seen in table 6 . The detector acceptance cut employed for the MINERvA CC0 $\pi 1 \mathrm{p}$ selection of events is observed as a change of slope around $q^{0}=0.6 \mathrm{GeV}$, clearly visible in the $1 \mathrm{p} 1 \mathrm{~h}$ distribution. In the case of $\mathrm{T} 2 \mathrm{~K}$, the hard cutoff is not visible and the distributions of momentum and energy transfers are narrower and shifted towards lower values, with smaller contamination from Res and DIS-Others components (see also table 6). In figure 27, one can also observe a shift in the value of $|\vec{q}|$. Actually, the mean value of $|\vec{q}|$ in the MINERvA (T2K) $1 \mathrm{p} 1 \mathrm{~h}$ component moves from $0.69 \mathrm{GeV}(0.61 \mathrm{GeV})$ in the $\mathrm{CC} 0 \pi$ data-sample to $0.77 \mathrm{GeV}(0.73 \mathrm{GeV})$ in the $\mathrm{CC} 0 \pi 1 \mathrm{p}$ one. This is a consequence of requesting a proton above $0.45 \mathrm{GeV}$ in the detector. 

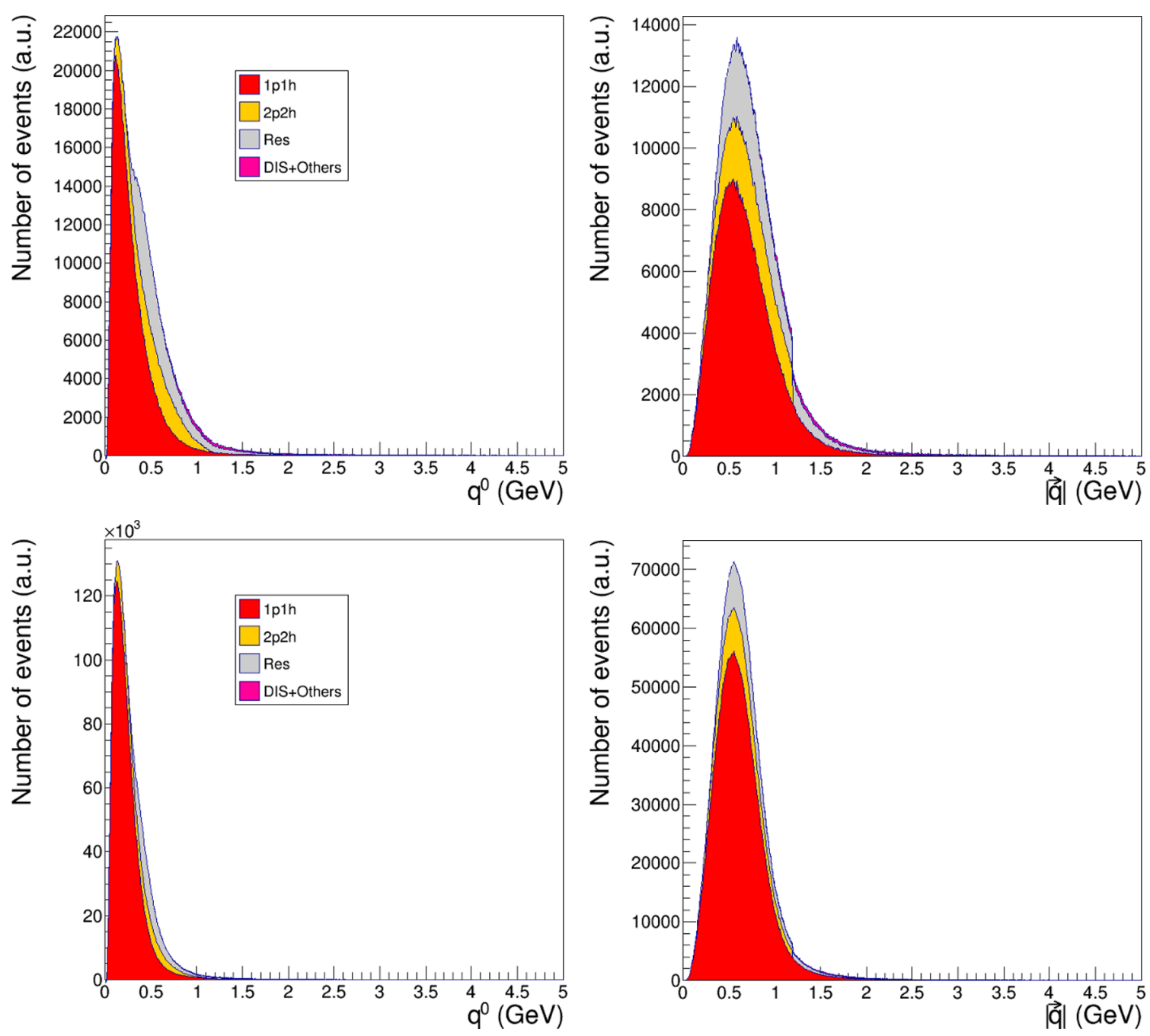

Figure 26. The same as figure 25 for $\mathrm{CC} 0 \pi$ selections.

Finally in figures 28 and 29, we show for the MINERvA CC0 $\pi$ sample, the $q^{0}$ and $|\vec{q}|$ distributions as a function of $p_{\perp}$ for the same $p_{\|}$binning as in figure 6 . A high occupancy region corresponding to the $\mathrm{CC} 1 \mathrm{p} 1 \mathrm{~h}$ contribution is clearly observed. A second one, mainly due to CC2p2h and CCRes events, is also visible in $q^{0}$, see figure 28 (note the logarithm scale in the $z$-coordinate). This second enhanced region is less visible in $|\vec{q}|$, see figure 29 , except for the low longitudinal momentum bins. The distributions also show that $|\vec{q}|$ values are similar for a given $p_{\perp}$ independently of the longitudinal momentum except for the case with $p_{\|}<3 \mathrm{GeV}$. The main differences between data and $\mathrm{MC}$ observed in figure 6 as function of $p_{\|}$are most probably caused by the CCRes and CC2p2h large $q^{0}$ contributions. 

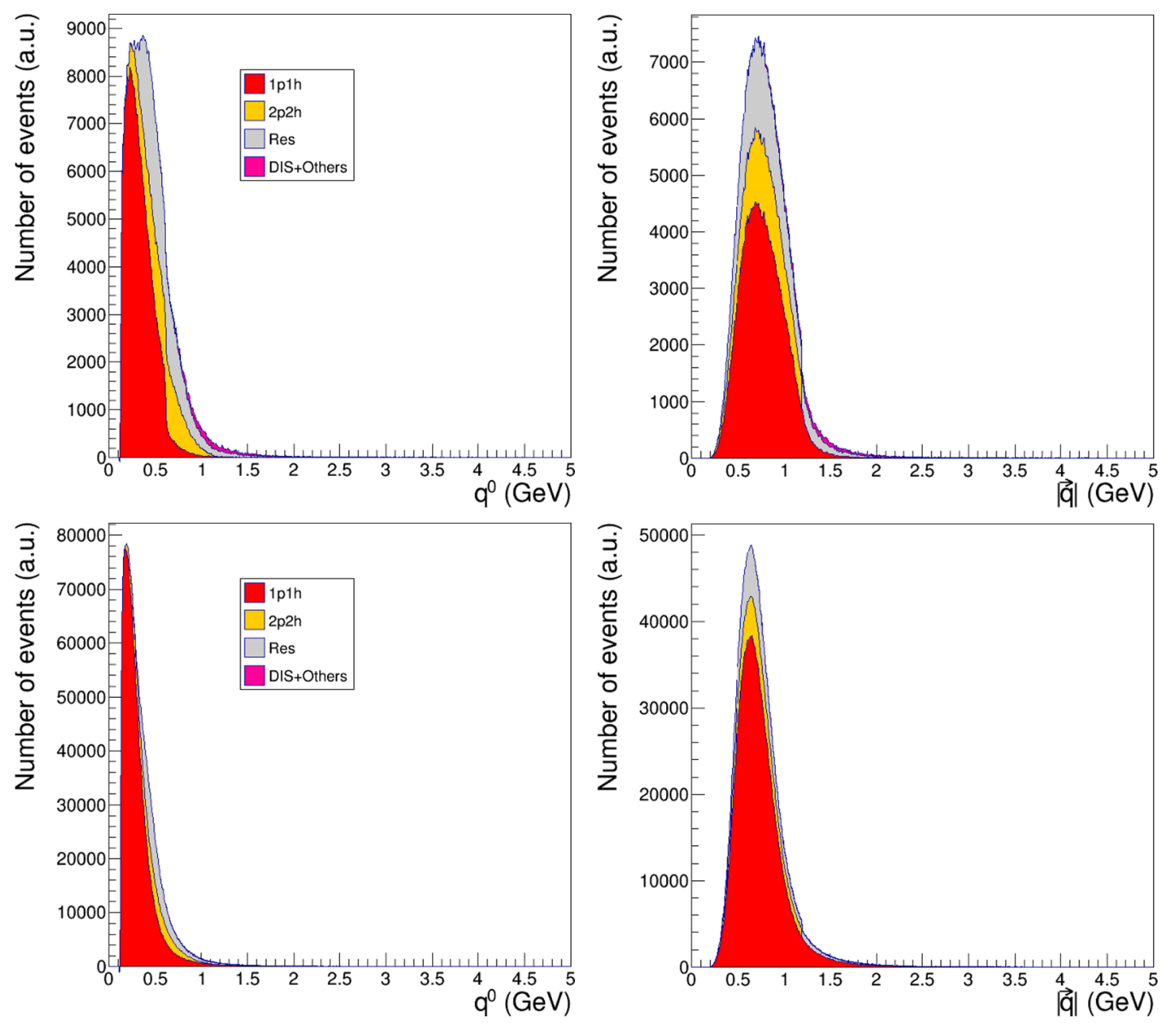

Figure 27. The same as figure 25 for $\mathrm{CC} 0 \pi 1 \mathrm{p}$ selections. 

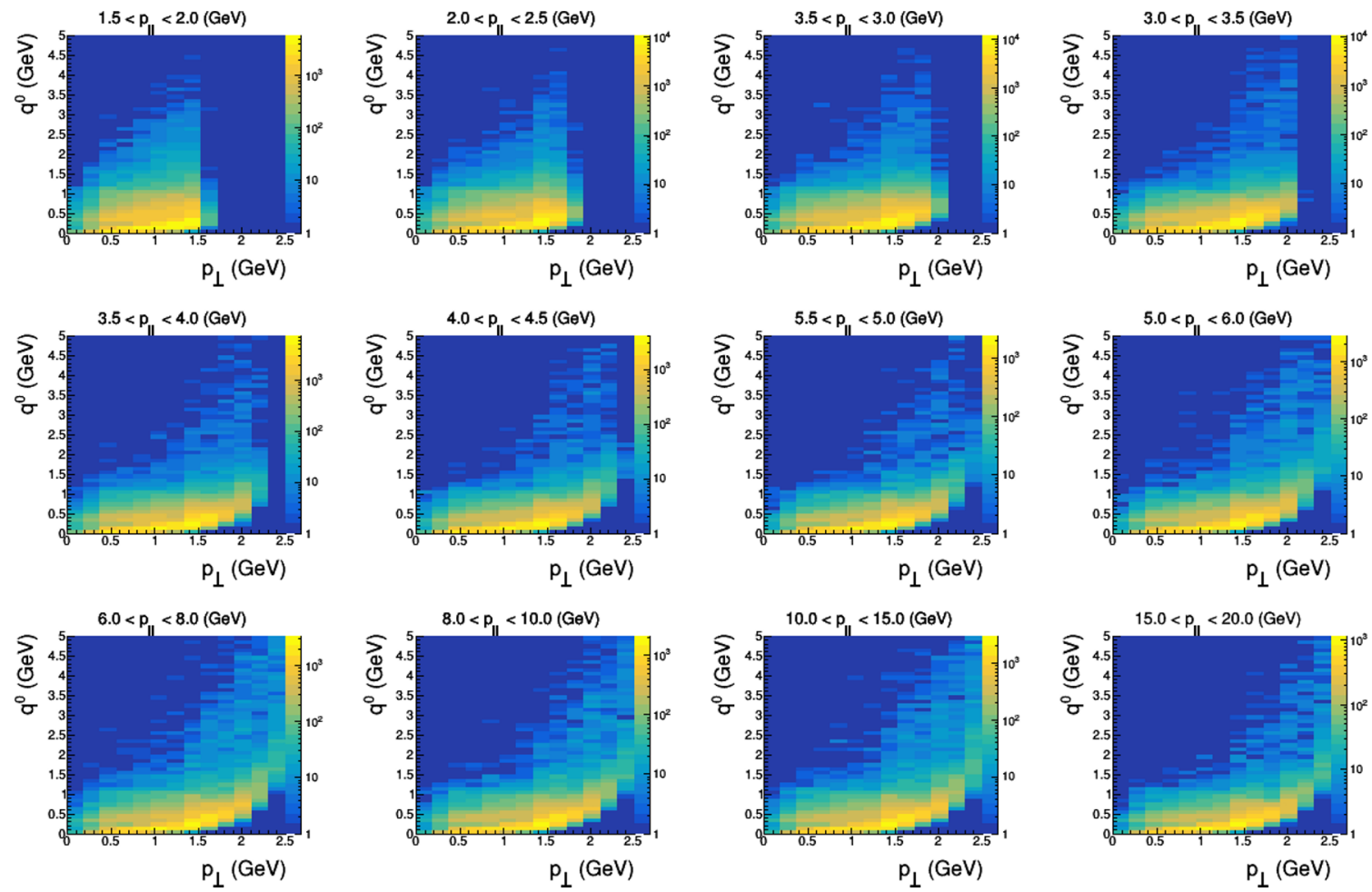

Figure 28. $q^{0}$ vs $p_{\perp}$ for MINERvA CC0 $\pi$ sample for the same $p_{\|}$bins as in figure 6 . The color code represents the number of events in arbitrary units. 

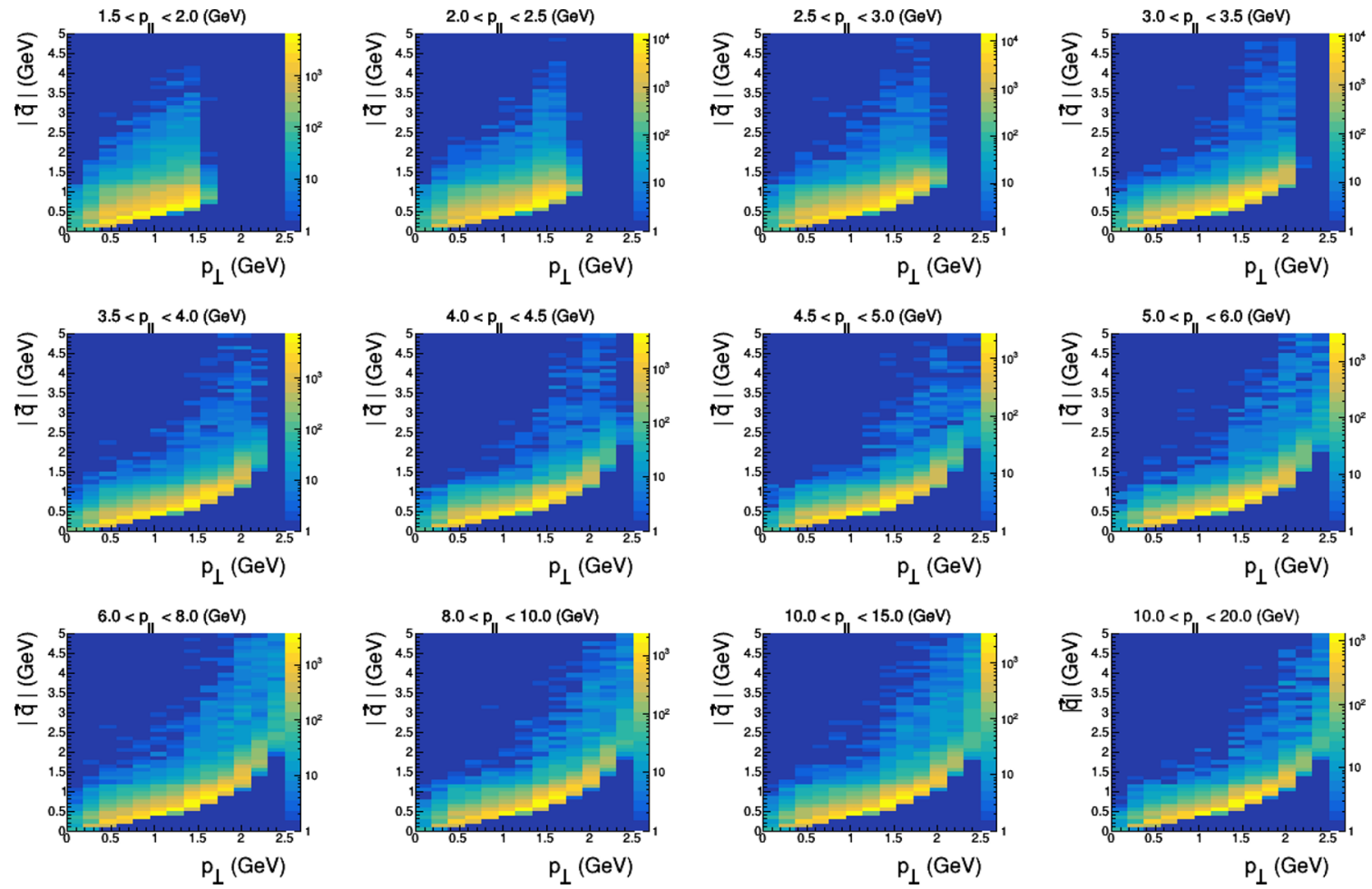

Figure 29. $|\vec{q}|$ vs $p_{\perp}$ for MINERvA CC0 $\pi$ sample for the same $p_{\|}$bins as in figure 6 . The color code represents the number of events in arbitrary units. 


\section{Acknowledgments}

This work was supported by the Swiss National Foundation Grant No. 200021_85012, the Spanish Ministerio de Economía y Competitividad and the European Regional Development Fund under contract FIS2017-84038-C2-1-P, the EU STRONG-2020 project under the program H2020-INFRAIA-2018-1, grant agreement no. 824093 and by Generalitat Valenciana under contract PROMETEO/2020/023. We thank the MINERVA collaboration for letting us notice the publication of their new results.

Open Access. This article is distributed under the terms of the Creative Commons Attribution License (CC-BY 4.0), which permits any use, distribution and reproduction in any medium, provided the original author(s) and source are credited.

\section{References}

[1] NuSTEC collaboration, NuSTEC White Paper: Status and challenges of neutrino-nucleus scattering, Prog. Part. Nucl. Phys. 100 (2018) 1 [arXiv:1706.03621] [InSPIRE].

[2] Hyper-Kamiokande Working Group collaboration, A Long Baseline Neutrino Oscillation Experiment Using J-PARC Neutrino Beam and Hyper-Kamiokande, (2014) [arXiv: 1412.4673] [INSPIRE].

[3] T2K collaboration, T2K neutrino flux prediction, Phys. Rev. D 87 (2013) 012001 [Addendum ibid. 87 (2013) 019902] [arXiv:1211.0469] [INSPIRE].

[4] J. Nieves, F. Sanchez, I. Ruiz Simo and M.J. Vicente Vacas, Neutrino Energy Reconstruction and the Shape of the CCQE-like Total Cross Section, Phys. Rev. D 85 (2012) 113008 [arXiv:1204.5404] [INSPIRE].

[5] L. Álvarez-Ruso, Y. Hayato and J. Nieves, Progress and open questions in the physics of neutrino cross sections at intermediate energies, New J. Phys. 16 (2014) 075015 [arXiv: 1403.2673] [INSPIRE].

[6] J. Nieves, J.E. Amaro and M. Valverde, Inclusive quasi-elastic neutrino reactions, Phys. Rev. C 70 (2004) 055503 [Erratum ibid. 72 (2005) 019902] [nucl-th/0408005] [INSPIRE].

[7] J. Nieves, I. Ruiz Simo and M.J. Vicente Vacas, Inclusive Charged-Current Neutrino-Nucleus Reactions, Phys. Rev. C 83 (2011) 045501 [arXiv:1102.2777] [INSPIRE].

[8] Y. Hayato, A neutrino interaction simulation program library NEUT, Acta Phys. Polon. B 40 (2009) 2477 [INSPIRE].

[9] O. Benhar, A. Fabrocini and S. Fantoni, The Nucleon Spectral Function in Nuclear Matter, Nucl. Phys. A 505 (1989) 267 [InSPIRE].

[10] O. Benhar, A. Fabrocini, S. Fantoni and I. Sick, Spectral function of finite nuclei and scattering of GeV electrons, Nucl. Phys. A 579 (1994) 493 [INSPIRE].

[11] O. Benhar, N. Farina, H. Nakamura, M. Sakuda and R. Seki, Electron- and neutrino-nucleus scattering in the impulse approximation regime, Phys. Rev. D 72 (2005) 053005 [hep-ph/0506116] [INSPIRE].

[12] T2K collaboration, Measurement of inclusive double-differential $\nu_{\mu}$ charged-current cross section with improved acceptance in the T2K off-axis near detector, Phys. Rev. D 98 (2018) 012004 [arXiv: 1801.05148] [INSPIRE]. 
[13] T2K collaboration, Simultaneous measurement of the muon neutrino charged-current cross section on oxygen and carbon without pions in the final state at T2K, Phys. Rev. D 101 (2020) 112004 [arXiv:2004.05434] [INSPIRE].

[14] T2K collaboration, Measurement of the muon neutrino charged-current single $\pi^{+}$production on hydrocarbon using the T2K off-axis near detector ND280, Phys. Rev. D 101 (2020) 012007 [arXiv: 1909.03936] [INSPIRE].

[15] MINERvA collaboration, Measurement of final-state correlations in neutrino muon-proton mesonless production on hydrocarbon at $\left\langle E_{\nu}\right\rangle=3 \mathrm{GeV}$, Phys. Rev. Lett. 121 (2018) 022504 [arXiv: 1805.05486] [INSPIRE].

[16] MINERvA collaboration, Double-differential inclusive charged-current $\nu_{\mu}$ cross sections on hydrocarbon in MINERvA at $\left\langle E_{\nu}\right\rangle \sim 3.5 \mathrm{GeV}$, Phys. Rev. D 101 (2020) 112007 [arXiv:2002.12496] [INSPIRE].

[17] MINERvA collaboration, Measurement of Quasielastic-Like Neutrino Scattering at $\left\langle E_{\nu}\right\rangle \sim 3.5 \mathrm{GeV}$ on a Hydrocarbon Target, Phys. Rev. D 99 (2019) 012004 [arXiv: 1811.02774] [INSPIRE].

[18] J.E. Sobczyk, J. Nieves and F. Sánchez, Exclusive-final-state hadron observables from neutrino-nucleus multinucleon knockout, Phys. Rev. C 102 (2020) 024601 [arXiv:2002.08302] [INSPIRE].

[19] MINERvA collaboration, Nucleon binding energy and transverse momentum imbalance in neutrino-nucleus reactions, Phys. Rev. D 101 (2020) 092001 [arXiv:1910.08658] [INSPIRE].

[20] L.A. Harewood and R. Gran, Elastic hadron-nucleus scattering in neutrino-nucleus reactions and transverse kinematics measurements, arXiv:1906.10576 [INSPIRE].

[21] T2K collaboration, Characterization of nuclear effects in muon-neutrino scattering on hydrocarbon with a measurement of final-state kinematics and correlations in charged-current pionless interactions at T2K, Phys. Rev. D 98 (2018) 032003 [arXiv:1802.05078] [INSPIRE].

[22] D. Rein and L.M. Sehgal, Neutrino Excitation of Baryon Resonances and Single Pion Production, Annals Phys. 133 (1981) 79 [INSPIRE].

[23] D. Rein and L.M. Sehgal, Coherent piO Production in Neutrino Reactions, Nucl. Phys. B 223 (1983) 29 [INSPIRE].

[24] D. Rein and L.M. Sehgal, PCAC and the Deficit of Forward Muons in $\pi^{+}$Production by Neutrinos, Phys. Lett. B 657 (2007) 207 [hep-ph/0606185] [INSPIRE].

[25] M. Glück, E. Reya and A. Vogt, Dynamical parton distributions revisited, Eur. Phys. J. C 5 (1998) 461 [hep-ph/9806404] [INSPIRE].

[26] A. Bodek and U.K. Yang, Modeling neutrino and electron scattering cross-sections in the few GeV region with effective LO PDFs, AIP Conf. Proc. 670 (2003) 110 [hep-ex/0301036] [INSPIRE].

[27] L.L. Salcedo, E. Oset, M.J. Vicente-Vacas and C. Garcia-Recio, Computer Simulation of Inclusive Pion Nuclear Reactions, Nucl. Phys. A 484 (1988) 557 [InSPIRE].

[28] E.S. Pinzon Guerra et al., Using world charged $\pi^{ \pm}$-nucleus scattering data to constrain an intranuclear cascade model, Phys. Rev. D 99 (2019) 052007 [arXiv: 1812.06912] [InSPIRE].

[29] MINERvA collaboration, Identification of nuclear effects in neutrino-carbon interactions at low three-momentum transfer, Phys. Rev. Lett. 116 (2016) 071802 [Addendum ibid. 121 (2018) 209902] [arXiv: 1511.05944] [INSPIRE]. 
[30] MINERvA collaboration, Design, Calibration, and Performance of the MINERvA Detector, Nucl. Instrum. Meth. A $\mathbf{7 4 3}$ (2014) 130 [arXiv:1305.5199] [INSPIRE].

[31] T2K ND280 FGD collaboration, The T2K Fine-Grained Detectors, Nucl. Instrum. Meth. A 696 (2012) 1 [arXiv:1204.3666] [INSPIRE].

[32] C. Andreopoulos et al., The GENIE Neutrino Monte Carlo Generator, Nucl. Instrum. Meth. A 614 (2010) 87 [arXiv:0905.2517] [INSPIRE].

[33] X.G. Lu, D. Coplowe, R. Shah, G. Barr, D. Wark and A. Weber, Reconstruction of Energy Spectra of Neutrino Beams Independent of Nuclear Effects, Phys. Rev. D 92 (2015) 051302 [arXiv: 1507.00967] [INSPIRE].

[34] X.G. Lu et al., Measurement of nuclear effects in neutrino interactions with minimal dependence on neutrino energy, Phys. Rev. C 94 (2016) 015503 [arXiv:1512.05748] [INSPIRE].

[35] A.P. Furmanski and J.T. Sobczyk, Neutrino energy reconstruction from one muon and one proton events, Phys. Rev. C 95 (2017) 065501 [arXiv: 1609.03530] [INSPIRE].

[36] R. Gonzaléz-Jiménez, G.D. Megias, M.B. Barbaro, J.A. Caballero and T.W. Donnelly, Extensions of Superscaling from Relativistic Mean Field Theory: the SuSAv2 Model, Phys. Rev. C 90 (2014) 035501 [arXiv: 1407.8346] [INSPIRE].

[37] T.W. Donnelly and I. Sick, Superscaling of inclusive electron scattering from nuclei, Phys. Rev. C 60 (1999) 065502 [nucl-th/9905060] [INSPIRE].

[38] M.B. Barbaro, R. Cenni, A. De Pace, T.W. Donnelly and A. Molinari, Relativistic y-scaling and the Coulomb sum rule in nuclei, Nucl. Phys. A 643 (1998) 137 [nucl-th/9804054] [INSPIRE].

[39] T. Golan, C. Juszczak and J.T. Sobczyk, Final State Interactions Effects in Neutrino-Nucleus Interactions, Phys. Rev. C 86 (2012) 015505 [arXiv: 1202.4197] [InSPIRE].

[40] U. Mosel, Neutrino event generators: foundation, status and future, J. Phys. G 46 (2019) 113001 [arXiv: 1904.11506] [INSPIRE].

[41] MINERvA collaboration, Probing nuclear effects with neutrino-induced charged-current neutral pion production, Phys. Rev. D 102 (2020) 072007 [arXiv:2002.05812] [INSPIRE]. 